\title{
Article \\ Efficiency of Environmental Protection Expenditures in EU Countries
}

\author{
Alan Barrell 1,2,3,4,5,6,7,8,9, Pawel Dobrzanski 10,11,*(D), Sebastian Bobowski ${ }^{12}$, Krzysztof Siuda 11 (D) \\ and Szymon Chmielowiec ${ }^{9}$
}

check for updates

Citation: Barrell, A.; Dobrzanski, P.; Bobowski, S.; Siuda, K.; Chmielowiec S. Efficiency of Environmental Protection Expenditures in EU Countries. Energies 2021, 14, 8443. https://doi.org/10.3390/en14248443

Academic Editor:

Fernando Morgado-Dias

Received: 9 November 2021

Accepted: 7 December 2021

Published: 14 December 2021

Publisher's Note: MDPI stays neutral with regard to jurisdictional claims in published maps and institutional affiliations.

Copyright: (c) 2021 by the authors. Licensee MDPI, Basel, Switzerland. This article is an open access article distributed under the terms and conditions of the Creative Commons Attribution (CC BY) license (https:// creativecommons.org/licenses/by/ $4.0 /)$.
Cambridge Worldwide Associates, Cambridge CB2 8PW, UK; Alan@alanbarrell.com

2 Wivenhoe Park, University of Essex, Colchester CO4 3SQ, UK

3 Centre for Entrepreneurial Learning, Judge Business School, University of Cambridge, Cambridge CB2 1QA, UK

4 Ashcroft Business School, Anglia Ruskin University, Cambridge CB1 1PT, UK

5 Cambridge Rare Diseases Network, Cambridge CB22 5NE, UK

6 Aavagen Oy, 20750 Turku, Finland

7 School of Economics, Liaoning University, Shenyang 110036, China

8 China Future Creative Class (CFCC) Ltd., St. John's Innovation Centre, Cambridge CB4 0WS, UK

9 The Cambridge Learning Gateway, St. John's Innovation Centre, Cambridge CB4 0WS, UK; sz.chmielowiec@camlg.com

10 Minda de Gunzburg Center for European Studies (CES), Harvard University, Cambridge, MA 02138, USA

11 Department of Mathematical Economics, Wroclaw University of Economics and Business,

53-345 Wroclaw, Poland; krzysztof.siuda@ue.wroc.pl

12 Department of International Economic Relations, Wroclaw University of Economics and Business, 53-345 Wroclaw, Poland; sebastian.bobowski@ue.wroc.pl

* Correspondence: pawel.dobrzanski@ue.wroc.pl or pdobrzanski@fas.harvard.edu

\begin{abstract}
Environmental protection policy is a widely discussed issue in scientific works. However, special attention should be also paid to the effectiveness of expenditures on environmental protection, and this is the main goal of this paper. The countries of the European Union were selected for this analysis due to the fact that, in recent years, this region has become an informal world leader with respect to the implementation of policy measures in the field of environmental protection. For that reason, the data envelopment analysis methodology was used, which allows the calculation of input-output efficiency for the years 2005-2015. The analysis shows that, among the 30 analyzed countries, the most effective in environmental protection actions is Finland. The hypothesis that higher environmental protection expenditures does not result in better environmental results has been confirmed. Our analysis confirmed the problem of the deteriorating efficiency of environmental expenditures across the selected European Union Member States, caused by increases in spending. This research may contribute to the discussion on environmental protection policy design and its assessment, as well as environmental policy results measurement.
\end{abstract}

Keywords: environmental protection expenditures; DEA methodology; relative efficiency; EU environmental policy

\section{Introduction}

Climate change and pollution are among the most serious challenges facing the world today. Therefore, in March 2007, the EU Heads of State and Government endorsed the European Commission's integrated strategy on climate change and energy, which sets out the EU's proposals for a global and comprehensive agreement to combat climate change after 2012, when the targets under the Kyoto Protocol expire. In Paris in December 2015, 195 countries (practically the whole world) agreed to the first-ever legally binding global deal to tackle climate change. In the EU and around the world, governments, companies and individuals are already working to tackle its causes and adapt to the changes it brings. Environmental protection expenditure are related to preventing, reducing and eliminating 
pollution and any other degradation of the environment, and in EU-27, they increased from 2006 to 2019 by $34 \%$.

However, environmental protection expenditures used to be neglected as a category of public expenditures in favor of, mainly, education, social security and healthcare. On the other hand, however, environmental protection fits perfectly into the category of public goods due to its shared consumption and gains, as well as its limited spectrum of exceptions, related to, among others, water- or land-based assets [1]. Expenditures on environmental protection address market failures with respect to externalities related to the environment [2], which are considered important determinants of environmental quality [3-8]. Depending on the set of pollutants considered and environmental components, i.e., the water, air or land to be impacted, jurisdiction can be either local, regional or global. Considering the fact that public goods are underprovided in a market economy, as classic public finance theory states, the institution of the state is expected to deliver them, even though that can be inefficient when compared to the provision of public goods by private sector. In fact, in the case of environmental protection expenditures, the role of the private sector is more and more substantial, resulting from the characteristics of environmental regulations that set some standards or enhance the self-regulation of the corporate sector. Importantly, there is a trend toward the privatization of utilities such as water and energy, which enhances the recategorization of some public environmental protection expenditures as private [1]. The empirical evidence of the impact of privatization on environmental protection expenditures is at least ambiguous to date. Recent studies by Xing and Tan [9] indicated that, in the case of high marginal environmental damage, the impact of privatization on social welfare results in the environmental policy of public enterprises, as well as environmental tax rates. An increase in environmental protection expenditures and the improvement of the environment due to privatization proved to be more probable if the public corporate sector is less environmentally conscious. Conversely, with a stronger focus on environmental protection within state-owned enterprises, privatization may reduce environmental protection expenditures and, as a consequence, the quality of the environment. Those results, however, are highly diverse, depending on the level of development of the country and the industry/sector of the economy [10-14].

The main goal of the paper is to evaluate if environmental protection expenditures are used efficiently. Our research fills the gap in the empirical literature studied in Section 2, as we address the efficiency of environmental protection expenditures. Literature review, covering numerous scientific papers, indicates that environmental protection policy and expenditures are widely discussed issues, whereas the efficiency of expenditures on environmental protection has been a niche topic so far. The study presented in this article verifies the hypothesis that higher environmental protection expenditures do not result in better environmental results. The EU countries were selected for this analysis due to the fact that, in recent years, this region has become an informal world leader with respect to the implementation of policy measures in the field of environmental protection laws [15], especially after the withdrawal of former US president Trump from the Paris Agreement [16]. Fortunately, newly elected US president Biden announced a return to this pact in 2021. Environmental conservation and pollution prevention across the Member States fit into the EU's general strategy of green transformation under the European Green Deal, i.e., withdrawal from fossil fuels, which, in turn, translates into a reduction of emissions resulting from fossil fuel combustion and a reduction in dependence on external energy supplies. Energy policy cannot be considered in isolation from the environmental policy of the EU [17]. This complies with Allen et al. [18], who stressed the significant impact of energy policy on environmental protection and expenditures. The environmental policy of the EU, as a sectoral policy that relies on the division of competencies between the EU institutions and member states, has gained a lot of importance in recent years, which has translated into regulatory progress [19] and financial allocations in the field of climate policy, which actually links the environmental and energy policies of the EU. However, the climate and energy policies of the EU have become highly politicized since 
the global financial crisis 2007-2009 [20]. Among the key legislative initiatives of the EU addressing environmental policy, there were: the Lisbon Strategy (2000), Emission Trading Scheme (2005), Lisbon Treaty (2007), Climate and Energy Package (2009), Europe 2020 Strategy (2010), and European Green Deal (2019). Under the European Green Deal, the EU is expected to achieve carbon neutrality by 2050 , which means net-zero greenhouse gases emissions, whereas by 2030 the EU commits itself to reducing those emissions by at least $55 \%$, to increase the shares of renewable energy in gross final energy consumption up to $32 \%$ and to increase the ratio of energy efficiency up to $32.5 \%$ [17,21-25]. One of the flagship initiatives of the European Green Deal is the New EU Forest Strategy for 2030. Among the key priorities, there is the promotion of sustainable forest bioeconomy, the improvement of the quantity and quality of the EU forests and the re- and afforestation of biodiverse forests. Under this strategy, forests may significantly contribute to the reduction of greenhouse gas emissions by 2030 by $55 \%$-according to estimations, up to 310 million tons of $\mathrm{CO}_{2}$. The protection of existing forest areas and the planting of new ones (the EU pledges to plant 3 billion new trees) may, therefore, support actions against climate change, as well as several important nature-based economic sectors, i.e., agriculture, construction and food and drink. Forest policy is expected to positively impact the seafood and insurance industries, when considering, for instance, the conservation of marine stocks and the protection of coastal wetlands. The EU Member States are either high- or middle-income countries, more or less experienced with respect to reindustrialization and the resulting reorientation toward environmental-friendly industries, technologies and energy resources [2]. Thus, studies on the efficiency of environmental expenditures enable the formulation of recommendations in the name of the rationalization of the EU- and national-level sectoral policies in this field.

The rest of the paper is organized as follows. Section 2 presents a review of the literature regarding environmental protection expenditures studies. Section 3 describes methodology. Section 4 presents the data, results and discussion. Section 5 concludes this research.

\section{Literature Review}

The hypothesis of environmental Kuznets curve for environmental protection expenditures states that, at early stage, of economic transition, the quality of the environment tend to deteriorate but improves prospectively due to structural transformation and the rising expectations of the society resulting from the increase in income per capita [26]. Lopez et al. [5], next to income level, stressed the role of scale, composition and technique, with special regard to higher labor efficiency and increased human-capital-intensive activities, as determinants of government expenditures' impact on environmental pollution. Rising public expenditures result in higher redistributive transfers, which translate into increased income equality, then, higher expectations with respect to the quality of the environment [27]. In this regard, government expenditures are necessary to combat inequality if development in itself proves insufficient [28]. On the other hand, market forces do not provide effective solutions to inequality; therefore, there is a need for state activity [29]. This, in turn, translates into policy measures established by the advanced institutions that used to be scarce within the developing world [30-33]. The demand for environmental quality, when treated as luxury public good, can be satisfied through government expenditures when other public goods are already provided [3,4]. Environmental expenditures are expected to decline as the economy reorients itself to more environmentally friendly industries, technologies and energy resources. This, however, might result in the relocation of dirty industries from higher- to lower-income countries [34], while the accumulation of capital in the former group of countries may serve as a trigger for the development of environmental-friendly technologies. A reduction in government size may deteriorate the prosperity of the country $[35,36]$. Considering the indirect effect of the reduction of government expenditures on environmental protection through its impact on income, the reduction of the government size in developing countries may result in 
the deterioration of the quality of the environment [6]. Thus, the reduction of government expenditures in developing countries should be accompanied by the introduction of environmental regulations and standards, including the ratification of international environmental treaties. Contrarily, in the case of higher-income countries, i.e., the selected EU Member States, authors verified empirically that cutting government expenditures resulted in an improvement in both income and environmental quality, especially in the long-run [27].

Importantly, empirical studies on the environmental Kuznets curve hypothesis, i.e., [1,16,37-72], have delivered at least ambiguous results with respect to the correlation between the level of income per capita and environmental pollution, especially when considering one of the most important greenhouse gases contributing to climate change, i.e., carbon dioxide. This greenhouse gas accounting for more than 75 percent of total emissions resulting from human activities has been adopted as a measure of environmental degradation in the empirical verification of the environmental Kuznets curve hypothesis $[58,64]$. Carbon dioxide emissions were proven to increase due to financial development, as pointed out by, i.e., Pata [66] and Shahbaz et al. [73], as well as industrialization and urbanization processes, as analyzed by, i.e., Liu and Bae [74]. The reduction of carbon dioxide can be achieved through the adoption of fiscal expenditures aimed at the development of a low-carbon economy, improvement in energy efficiency and the optimization of energy structure $[5,75,76]$. From the global perspective, the impact of economic growth and energy structure, as well as population density, on regional and national carbon dioxide emission was studied by, among others, Hubacek et al. [77] and Chen et al. [78]; and from the national perspective, by Zhang et al. [79] and Chen et al. [78]; and from the micro-perspective by Cole et al. [80] and Xu et al. [81]. Whereas Omri et al. [82] and Wang et al. [83] adopted econometric modelling, Rhee et al. [84] and Cansino et al. [85] used input-output methods, and Nag and Parikh [86] and Tan et al. [87] used a factor decomposition approach.

Analyses dedicated to environmental expenditures in the context of carbon dioxide emissions and GDP were conducted for 19 African countries [54], China and India [62], Croatia [60], Malaysia [88], India [64], Turkey [66], Azerbaijan [89] and Eastern European countries [68]. Empirical studies on developed countries addressed the United States [58], Portugal and Spain [61] and the EU countries [44,47,49,50,52,53,56,70,90-93]. Several authors, Panayotou [94], Cole and Elliott [95], Dinda [96] and Tsurumi and Managi [97], analyzed the correlation between carbon dioxide emissions and trade liberalization processes in the context of the environmental Kuznets curve hypothesis. On the other hand, the empirical verification of the impact of renewable energy sources on ecological footprint, which is considered in our analysis next to carbon dioxide with respect to the efficiency of environmental expenditures, were studied across the EU Member States only by a few authors, i.e., Destek et al. [16], Menegaki and Tsagarakis [98].

Maneejuk et al. [70] has confirmed the environmental Kuznets curve hypothesis at the country-level only for 9 of 44 analyzed countries of the EU, OECD and G7 groupings. Interestingly, empirical studies by Pearce and Palmer [1], based on data from 1970s-1990s for OECD countries, pointed at no unambiguous upward trend with respect to the involvement of the private sector in environmental protection expenditures, with highly diversified country-level effects. Whereas several authors, i.e., Freeman [99,100], Hahn [101] and Portney [102], addressed the issue of the relative gains and costs of environmental regulations, there is relatively scarce literature on the budgetary costs of environmental protection in the context of government efficiency.

Ercolano and Romano [2] investigated environmental expenditures across 21 European countries (the selected EU Member States, United Kingdom, Iceland and Norway), using a sigma-convergence approach, followed by principal component and cluster analysis. As indicated by the authors, public environmental protection expenditures, as well as their composition within various initiatives and strategies, differ significantly across the countries. However, not only the level but also the efficiency of environmental expenditures should take into consideration the public spending in this field with environmental 
outcomes, such as greenhouse gas emissions and renewable energy development [103]. Due to the multidimensional character of public expenditures' impacts, it is useful to adopt composite indicators, such as the environmental performance index (EPI) for the purposes of policy analysis [104]. The fact is that EPI serves as an aggregate measure of the results of policies aimed at the improvement of environmental quality, built on measurable outcomes such as emissions or deforestation, instead of policy inputs, i.e. public expenditures programs. Ercolano and Romano [2] identified no significant bivariate correlation between EPI, public environmental expenditures in terms of GDP and GDP per capita. Authors denied the hypothesis of the convergence of public environmental expenditures across the European countries, with no important changes in the composition of spending in this field. This complied with results obtained by Apergis et al. [105], according to which there is no convergence across the EU Member States, irrespective of several global- and regional-level directives and strategies dedicated to environmental protection, i.e., Sustainable Development Goals, Paris Agreement, Europe 2020 Strategy and the European Green Deal. In fact, a study by Ercolano and Romano [2] proved to be one of the first empirical contributions to the academic debate on the efficiency of environmental expenditures by verifying a correlation between the size and composition of public environmental expenditures and selected environmental performances and results.

Empirical findings by Apergis et al. [105] proved to be in line with analyses by Ferreiro et al. [106,107], which actually pointed at divergence across the EU Member States with respect to the size and composition of environmental expenditures. In fact, there is no single model of fiscal policy that enables the boosting of economic growth, prosperity and social welfare, including environmental quality that impacts people's life assessment from country to country.

Halkos and Paizanos [6] investigated the public environmental expenditures of 77 countries in the years 1980-2000 and their direct and indirect impacts on the income level, as well as the impacts of income level on environmental pollution. Methodology was based on econometric modelling with panel analysis. The authors focused on the emissions of carbon dioxide and sulfur dioxide, indicating that, in the case of carbon dioxide, government spending had an insignificant direct impact on emissions per capita but a negative one with respect to indirect effects. The authors concluded that the reduction of government expenditures in higher-income countries increases both income and environmental quality, as already established environmental regulations and standards would diminish returns from further increases in government size. This, in turn, provides some analogy to the environmental Kuznets curve hypothesis.

Empirical analysis by Ullah et al. [108] considered the asymmetrical effects of fiscal policy on the quality of the environment across the ten Asian countries responsible for the largest carbon emissions, including China and India, as well as Turkey, Iran and Japan, in the years 1981-2018. The authors, using a non-linear autoregressive distributed lag approach, identified the negative impact of a positive shock in government expenditure on environmental quality in the case of, among others, China, India, Turkey and Thailand, while only in the case of Japan did it prove to be positive. On the other hand, only in the case of Japan did the quality of the environment deteriorate in the studied period, because of the reduction in government expenditures. Moreover, due to an increase in governmental income tax revenues, there was an increase in government expenditures, which, in turn, increased carbon emissions everywhere except for Japan, again. As argued by the authors, short-run asymmetric effects translated into long-run effects across most of the Asian countries.

Recently, several analyses dedicated to the impacts of environmental expenditures and their efficiency focused on China, the largest contributor to global carbon dioxide emissions. After nearly three decades of dynamic economic growth, China faces the challenges of structural adjustments and environmental efficiency $[109,110]$. Zhou and Zhang [111], using a data envelopment analysis (DEA) method, investigated the efficiency of fiscal policies aimed at environmental pollution control across 30 Chinese provinces 
in the years 2007-2017. Authors empirically proved an improvement in this field since 2014, with special regard to the eastern regions of the country. Analysis confirmed that the efficiency of fiscal policy in the environmental field was negatively impacted by fiscal expenditure decentralization, while in the case of fiscal revenue decentralization, this effect proved to be non-significant. It was recommended to increase public expenditures for environmental pollution control, as well as for the central transfer payment system for environmental protection.

\section{Methods and Data}

Data envelopment analysis (DEA), created by Farrell [112] and developed by Charnes et al. [113], is the main methodology used in this article. DEA allows the calculation of the maximum performance value for each decision-making unit (DMU) relative to all DMUs in the study. DEA methodology constructs the efficiency production frontier and distance from the frontier at the nearest point for each DMU [114]. It should be noted that DEA measures relative technical efficiency, and, therefore, efficient DMUs are bestpractice frontiers rather than production frontiers [115]. Thus, DEA requires the careful interpretation of results. In DEA, variables that may duplicate information should be removed from the model [116].

DEA methodology can be divided into input- and output-oriented models. An inputoriented model assumes a proportional reduction in input, while outputs remain unchanged. By analogy, an output-oriented model undertakes a proportional increase in outputs, while the proportion of inputs remains unchanged [117]. Another division of the DEA methods is based on constant or variable returns to scale (CRS or VRS).

DEA calculates efficiency as the ratio of the weighted sum of outputs to the weighted sum of inputs [118]. The CRS model maximizes this ratio (see Equations (1)-(3)) [119]:

$$
\operatorname{Max} \theta_{0}=\frac{\sum_{\mathrm{r}=1}^{\mathrm{s}} \mathrm{u}_{\mathrm{r}} \mathrm{y}_{\mathrm{rk}}}{\sum_{\mathrm{i}=1}^{\mathrm{m}} \mathrm{v}_{\mathrm{i}} \mathrm{x}_{\mathrm{ik}}}
$$

subject to:

$$
\begin{gathered}
\frac{\sum_{r=1}^{\mathrm{s}} \mathrm{u}_{\mathrm{r}} \mathrm{y}_{\mathrm{rj}}}{\sum_{\mathrm{i}=1}^{\mathrm{m}} \mathrm{v}_{\mathrm{i}} \mathrm{x}_{\mathrm{ij}}} \leq 1, \\
\mathrm{u}_{\mathrm{r}}, \mathrm{v}_{\mathrm{i}} \geq 0 ; \forall \mathrm{r}=1, \ldots, \mathrm{s} ; \mathrm{i}=1, \ldots, \mathrm{m} ; \mathrm{j}=1, \ldots, \mathrm{n}
\end{gathered}
$$

where:

$\mathrm{u}_{\mathrm{r}}$-weight of output

$\mathrm{v}_{\mathrm{i}}$-weight of input

$\mathrm{y}_{\mathrm{rj}}$ - output

$\mathrm{x}_{\mathrm{ij}}$-input

While VRS model use supplementary restriction equation [113]:

$$
\sum_{j=1}^{n} \lambda_{j}=1
$$

where $\lambda$ is the corresponding solution vector for the optimization, and $\mathrm{n}$ is the number of DMUs.

This linear programming problem can be solved according to two different approaches. In the first, weighted sums of outputs are maximized while keeping inputs constant (outputoriented model). In the second, the weighted sums of inputs are minimized while keeping the outputs constant (input-oriented model). The primary equations for each model, known as the multiplier form, are given below [120].

The CRS input-oriented model equation becomes: 
Subject to

$$
\begin{gathered}
y_{r k}-\sum_{j=1}^{n} \lambda_{j} y_{r j} \leq 0 ; r=1, \ldots, s \\
\theta_{k} x_{i k}-\sum_{j=1}^{n} \lambda_{j} x_{i j} \geq 0 ; i=1, \ldots, m \\
\lambda_{j} \geq 0 ; \forall j=1, \ldots, n
\end{gathered}
$$

The VRS input-oriented model equation becomes:

$$
\text { Minimize } \theta_{\mathrm{k}}
$$

Subject to

$$
\begin{gathered}
y_{r k}-\sum_{j=1}^{n} \lambda_{j} y_{r j} \leq 0 ; r=1, \ldots, s \\
\theta_{k} x_{i k}-\sum_{j=1}^{n} \lambda_{j} x_{i j} \geq 0 ; i=1, \ldots, m \\
\sum_{j=1}^{n} \lambda j=1 \\
\lambda_{j} \geq 0 ; \forall j=1, \ldots, n
\end{gathered}
$$

There are numerous advantages to using DEA methodology. DEA can be used when the relationship between variables does not have a known mathematical function [121]. DEA is a flexible method that can be adapted to the data [122]. Moreover, multiple input and output variables in different units can be analyzed at the same time, without a priori information about the importance of particular variables [123]. Furthermore, the sources of inefficiency for analyzed units can be analyzed [117]. There are also some limitations. DEA does not analyze qualitative variables. The inclusion of a large number of input and output variables would reduce the discriminant power of DEA. Also, DEA is criticized for overestimating efficiency and providing information about dominant DMUs [121]. There is also a noticeable negative correlation between efficiency and the number of DMUs [124].

The presented research has some limitations. Firstly, the selected input and output variables and research periods were limited by the availability of international statistics. There were no available more up-to-date data for environment statistics for all analyzed countries. The available data was mainly until 2017; the latest statistics are missing in generally available databases. Only basic statistics for a selected group of countries is available. Some data, such as water pollution, are incomplete or unavailable for all years and countries, and therefore, they had to be removed from the model. Consequently, the analysis would be more complex if this study analyzed additional indicators, such as the effects of all actions covered by environmental protection expenditures, i.e., the protection of ambient air and climate; wastewater management; waste management; the protection and remediation of soil, groundwater and surface water; noise and vibration abatement; protection of biodiversity and landscapes; protection against radiation; research and development and other environmental protection activities. However, a lack of data in some statistics prevented us from creating a comparable dataset. The possibility of using the model aggregate input variable with specific output variables is one of the main advantages of DEA methodology, which allows us to calculate effectiveness without specifying the mathematical form of the production function. On the other hand, this might be treated as one of the limitations of our study. Introducing to the model all possible combinations of specific inputs and outputs would provide a more general picture of environmental conditions in the analyzed countries; however, simultaneously, it would cause a reduction in the discriminatory power of the DEA. Adding variables to a DEA model will result in 
higher efficiency scores and an expanded set of efficient DMUs. Therefore, in our analysis, we have followed the principle that the number of variables should be less than one-third of the DMUs. In this research, the DEA assessment was performed for the selected group of 30 countries. Expanding the research objects may cause effective DMUs to become ineffective comparing to the new object group. The analysis of a more extended period could bring more general conclusions and recommendations for environmental policies. An additional challenge is the correctness of the environmental outcomes and the indication of the proper time lag between inputs and outputs. In our study, there were no abrupt changes in spending in the EU countries. If they were increasing, they were increasing proportionally. Thanks to this, we would obtain very similar results if we waited, for example, two or three years for the effects. It is also worth noting that the effects are faster in some countries than in others. Therefore, the authors adopted the principle that they would not use time-lag. Our analysis does not take into account differences in energy demand or the favorability of the conditions and capabilities of countries to invest in renewable energy. On the one hand, northern European countries have better natural conditions for renewable energy production, in particular wind and water power, but on the other hand, due to climate, their demand for energy is higher. Southern European countries have warmer climate, as a consequence, demand less electricity and enjoy better conditions for solar energy production. We need to highlight also that the selected research period includes the subprime crisis, which caused an economic downturn in several EU countries and, consequently, lower funding for environmental protection.

In our research, diagnostic variables were selected based on available data from the Eurostat and World Bank. Input indicators are environmental protection expenditures (as \% of GDP), represented by EPE. Expenditure for environmental protection consists of outlays and other transactions related to:

- inputs for environmental protection activities (energy, raw materials and other intermediate inputs, wages and salaries, taxes linked to production, the consumption of fixed capital);

- capital formation and the buying of land (investment) for environmental protection activities;

- users' outlays for buying environmental protection products;

- transfers for environmental protection (subsidies, investment grants, international aid, donations, taxes earmarked for environmental protection, etc.).

More than half of the environmental protection expenditure is incurred by corporations, the rest by the general government, non-profit institutions serving households and households. However, it is worth mentioning that corporations and households environmental expenditures are directly and indirectly derived from government-imposed regulations and rules.

The three output indicators chosen for analysis were as follows: (a) forest area, (b) renewable electricity output, (c) $\mathrm{CO}_{2}$ emissions. The below principle was followed with respect to the selection of variables [125].

$$
\mathrm{n} \geq 3(\mathrm{~s}+\mathrm{m})
$$

where:

$\mathrm{s}$-number of inputs

$\mathrm{m}$-number of outputs

$\mathrm{n}$-numbers of DMUs

Table 1 presents a set of variables. In the DEA method, a positive relationship between inputs and outputs is assumed. Therefore, as in our case, the non-desirable out- 
put of $\mathrm{CO}_{2}$ emissions (destimulant) was transformed into desirable (stimulant) with the following transform:

$$
X_{i j}^{S}=1-\frac{X_{i j}^{D}}{\max _{i} X_{i j}^{D}}
$$

Table 1. Indicators and Sources.

\begin{tabular}{|c|c|c|c|}
\hline Variable & Indicator Name & Units & Source \\
\hline Input-EPE & Environmental protection expenditure & $(\%$ GDP) & Eurostat, 2021 [126] \\
\hline Output $1-$ FA & Forest area & (\% of land area) & World Bank, 2021 [127] \\
\hline Output $2-\mathrm{REO}$ & Renewable electricity output & (\% of total electricity output) & World Bank, 2021 [127] \\
\hline Output $3-\mathrm{COE}$ & $\mathrm{CO}_{2}$ emissions & (metric tons per capita) & World Bank, 2021 [127] \\
\hline
\end{tabular}

\section{Results and Discussion}

Calculations for the purposes of the examination of the relationship between environmental protection expenditures and environmental results were performed in DeaFrontier Software. The research covers 30 European countries and the period from 2005 to 2015. Due to the limitation of article length, authors present only the sample data for the input and output indicators for one year, 2015, in Table 2.

Table 2. Diagnostic set of input and output data-European countries in 2015. Source: World Bank [127], Eurostat [126].

\begin{tabular}{|c|c|c|c|c|c|}
\hline 2015 & Country/Indicators & EPE & FA & REO & $\mathrm{COE}$ \\
\hline 1 & AUT & 0.40 & 46.88 & 76.49 & 0.56 \\
\hline 2 & BEL & 1.30 & 22.76 & 20.80 & 0.46 \\
\hline 3 & BGR & 0.80 & 35.22 & 17.99 & 0.62 \\
\hline 4 & $\mathrm{CHE}$ & 0.60 & 31.73 & 62.20 & 0.73 \\
\hline 5 & CYP & 0.40 & 18.69 & 8.78 & 0.67 \\
\hline 6 & CZE & 1.10 & 34.54 & 11.40 & 0.42 \\
\hline 7 & DEU & 0.60 & 32.73 & 29.23 & 0.45 \\
\hline 8 & DNK & 0.40 & 14.58 & 65.51 & 0.67 \\
\hline 9 & ESP & 0.90 & 36.86 & 34.95 & 0.67 \\
\hline 10 & EST & 0.70 & 51.35 & 14.42 & 0.23 \\
\hline 11 & FIN & 0.20 & 73.11 & 44.50 & 0.52 \\
\hline 12 & FRA & 1.00 & 31.03 & 15.86 & 0.72 \\
\hline 13 & GBR & 0.80 & 13.00 & 24.84 & 0.62 \\
\hline 14 & GRC & 1.50 & 31.45 & 28.66 & 0.63 \\
\hline 15 & HRV & 0.70 & 34.35 & 66.83 & 0.75 \\
\hline 16 & HUN & 1.20 & 22.85 & 10.58 & 0.72 \\
\hline 17 & IRL & 0.40 & 10.95 & 27.97 & 0.53 \\
\hline 18 & ISL & 0.60 & 0.49 & 99.98 & 0.63 \\
\hline 19 & ITA & 0.90 & 31.61 & 38.68 & 0.68 \\
\hline 20 & LTU & 0.50 & 34.80 & 39.41 & 0.72 \\
\hline 21 & LVA & 0.70 & 53.97 & 50.17 & 0.78 \\
\hline 22 & MLT & 1.90 & 1.09 & 7.67 & 0.78 \\
\hline 23 & NLD & 1.40 & 11.16 & 12.44 & 0.37 \\
\hline 24 & NOR & 0.90 & 33.17 & 97.71 & 0.44 \\
\hline 25 & POL & 0.60 & 30.81 & 13.80 & 0.54 \\
\hline 26 & PRT & 0.60 & 34.74 & 47.53 & 0.71 \\
\hline 27 & ROU & 1.00 & 29.82 & 39.75 & 0.78 \\
\hline 28 & SVK & 0.90 & 40.35 & 22.68 & 0.64 \\
\hline 29 & SVN & 1.00 & 61.96 & 29.39 & 0.63 \\
\hline 30 & SWE & 0.40 & 68.92 & 63.26 & 0.76 \\
\hline
\end{tabular}

Note: AUT—Austria, BEL—Belgium, BGR—Bulgaria, CHE—Switzerland, CYP—Cyprus, CZE—The Czech Republic, DEU—Germany, DNK—Denmark, ESP—Spain, EST—Estonia, FIN—Finland, FRA—ZZZ, GBR—United Kingdom, BEL—Belgium, GRC—Greece, HRVCroatia, HUN-Hungary, IRL-Ireland, ISL-Iceland, ITA-Italy, LTU—Lithuania, LVA-Latvia, MLT_Malta, NLD—Netherlands, NOR-Norway, POL-Poland, PRT—Portugal, ROU—Romania, SVK-Slovak Republic, SVN—Slovenia. EPE—environmental protection expenditure ( $\%$ GDP), FA—forest area ( $\%$ of land area), $\mathrm{REO}$ - renewable electricity output ( $\%$ of total electricity output), $\mathrm{COE}-\mathrm{CO}_{2}$ emissions (metric tons per capita). 
An input-oriented model is used in the analysis, as most of the environmental protection strategies focus more on inputs than on outputs. The authors used both CRS and VRS models to avoid overestimation. As pointed out by [128,129], using only the VRS model may ignore subsets of proportional inputs and outputs. Therefore the authors used both the CRS and VRS models and then the average efficiency index. The results are presented in Tables 3 and 4.

Table 3. Input-Oriented CRS Efficiency of EPS in 2015. Source: Authors' calculations in DEAFrontier.

\begin{tabular}{|c|c|c|c|c|c|c|}
\hline DMU No. & DMU Name & Input-Oriented CRS Efficiency & $\sum \lambda$ & RTS & Benchmarks & DMU \\
\hline 1 & AUT & 0.859 & 1.719 & Decreasing & 1.719 & FIN \\
\hline 2 & BEL & 0.135 & 0.878 & Increasing & 0.878 & FIN \\
\hline 3 & BGR & 0.296 & 1.182 & Decreasing & 1.182 & FIN \\
\hline 4 & $\mathrm{CHE}$ & 0.469 & 1.410 & Decreasing & 1.410 & FIN \\
\hline 5 & CYP & 0.647 & 1.294 & Decreasing & 1.294 & FIN \\
\hline 6 & CZE & 0.146 & 0.800 & Increasing & 0.800 & FIN \\
\hline 7 & DEU & 0.289 & 0.867 & Increasing & 0.867 & FIN \\
\hline 8 & DNK & 0.736 & 1.472 & Decreasing & 1.472 & FIN \\
\hline 9 & ESP & 0.285 & 1.281 & Decreasing & 1.281 & FIN \\
\hline 10 & EST & 0.201 & 0.702 & Increasing & 0.702 & FIN \\
\hline 11 & FIN & 1.000 & 1.000 & Constant & 1.000 & FIN \\
\hline 12 & FRA & 0.276 & 1.378 & Decreasing & 1.378 & FIN \\
\hline 13 & GBR & 0.296 & 1.184 & Decreasing & 1.184 & FIN \\
\hline 14 & GRC & 0.161 & 1.208 & Decreasing & 1.208 & FIN \\
\hline 15 & HRV & 0.429 & 1.502 & Decreasing & 1.502 & FIN \\
\hline 16 & HUN & 0.231 & 1.385 & Decreasing & 1.385 & FIN \\
\hline 17 & IRL & 0.508 & 1.016 & Decreasing & 1.016 & FIN \\
\hline 18 & ISL & 0.749 & 2.247 & Decreasing & 2.247 & FIN \\
\hline 19 & ITA & 0.288 & 1.296 & Decreasing & 1.296 & FIN \\
\hline 20 & LTU & 0.554 & 1.386 & Decreasing & 1.386 & FIN \\
\hline 21 & LVA & 0.428 & 1.500 & Decreasing & 1.500 & FIN \\
\hline 22 & MLT & 0.157 & 1.488 & Decreasing & 1.488 & FIN \\
\hline 23 & NLD & 0.102 & 0.716 & Increasing & 0.716 & FIN \\
\hline 24 & NOR & 0.488 & 2.196 & Decreasing & 2.196 & FIN \\
\hline 25 & POL & 0.343 & 1.030 & Decreasing & 1.030 & FIN \\
\hline 26 & PRT & 0.452 & 1.357 & Decreasing & 1.357 & FIN \\
\hline 27 & ROU & 0.298 & 1.491 & Decreasing & 1.491 & FIN \\
\hline 28 & SVK & 0.275 & 1.237 & Decreasing & 1.237 & FIN \\
\hline 29 & SVN & 0.240 & 1.201 & Decreasing & 1.201 & FIN \\
\hline 30 & SWE & 0.729 & 1.458 & Decreasing & 1.458 & FIN \\
\hline
\end{tabular}

Note: DMU—decision-making unit, $\Sigma \lambda$ —Lambda sum, Unit, RTS—Returns to Scale, AUT—Austria, BEL-Belgium, BGR—Bulgaria, CHESwitzerland, CYP—Cyprus, CZE-The Czech Republic, DEU—Germany, DNK—Denmark, ESP—Spain, EST—Estonia, FIN—Finland, FRA-ZZZ, GBR-United Kingdom, BEL-Belgium, GRC—Greece, HRV—Croatia, HUN—Hungary, IRL-Ireland, ISL-Iceland, ITAItaly, LTU—Lithuania, LVA—Latvia, MLT—Malta, NLD—Netherlands, NOR—Norway, POL—Poland, PRT—Portugal, ROU—Romania, SVK-Slovak Republic, SVN-Slovenia.

Table 4. Input-Oriented VRS Efficiency of EPS in 2015. Source: Authors' calculations in DEAFrontier.

\begin{tabular}{|c|c|c|c|c|c|c|c|c|}
\hline DMU No. & DMU Name & Input-Oriented VRS Efficiency & Benchmarks & DMU & Lambda & DMU & Lambda & DMU \\
\hline 1 & AUT & 1.000 & 1.000 & AUT & & & & \\
\hline 2 & BEL & 0.154 & 1.000 & FIN & & & & \\
\hline 3 & BGR & 0.349 & 0.602 & FIN & 0.398 & SWE & & \\
\hline 4 & $\mathrm{CHE}$ & 0.640 & 0.032 & AUT & 0.079 & FIN & 0.888 & SWE \\
\hline 5 & CYP & 0.821 & 0.359 & FIN & 0.641 & SWE & & \\
\hline 6 & CZE & 0.182 & 1.000 & FIN & & & & \\
\hline 7 & DEU & 0.333 & 1.000 & FIN & & & & \\
\hline 8 & DNK & 0.941 & 0.336 & AUT & 0.117 & FIN & 0.547 & SWE \\
\hline 9 & ESP & 0.359 & 0.386 & FIN & 0.614 & SWE & & \\
\hline 10 & EST & 0.286 & 1.000 & FIN & & & & \\
\hline
\end{tabular}


Table 4. Cont.

\begin{tabular}{|c|c|c|c|c|c|c|c|c|}
\hline DMU No. & DMU Name & Input-Oriented VRS Efficiency & Benchmarks & DMU & Lambda & DMU & Lambda & DMU \\
\hline 11 & FIN & 1.000 & 1.000 & FIN & & & & \\
\hline 12 & FRA & 0.365 & 0.175 & FIN & 0.825 & SWE & & \\
\hline 13 & GBR & 0.350 & 0.599 & FIN & 0.401 & SWE & & \\
\hline 14 & GRC & 0.194 & 0.545 & FIN & 0.455 & SWE & & \\
\hline 15 & HRV & 0.598 & 0.007 & AUT & 0.095 & ISL & 0.898 & SWE \\
\hline 16 & HUN & 0.307 & 0.159 & FIN & 0.841 & SWE & & \\
\hline 17 & IRL & 0.518 & 0.964 & FIN & 0.036 & SWE & & \\
\hline 18 & ISL & 1.000 & 1.000 & ISL & & & & \\
\hline 19 & ITA & 0.366 & 0.354 & FIN & 0.646 & SWE & & \\
\hline 20 & LTU & 0.737 & 0.157 & FIN & 0.843 & SWE & & \\
\hline 21 & LVA & 1.000 & 1.000 & LVA & & & & \\
\hline 22 & MLT & 0.324 & 0.722 & LVA & 0.278 & SWE & & \\
\hline 23 & NLD & 0.143 & 1.000 & FIN & & & & \\
\hline 24 & NOR & 1.000 & 1.000 & - & & & & \\
\hline 25 & POL & 0.355 & 0.934 & FIN & 0.066 & SWE & & \\
\hline 26 & PRT & 0.593 & 0.222 & FIN & 0.778 & SWE & & \\
\hline 27 & ROU & 0.636 & 0.786 & LVA & 0.214 & SWE & & \\
\hline 28 & SVK & 0.337 & 0.482 & FIN & 0.518 & SWE & & \\
\hline 29 & SVN & 0.288 & 0.561 & FIN & 0.439 & SWE & & \\
\hline 30 & SWE & 1.000 & 1.000 & SWE & & & & \\
\hline
\end{tabular}

Note: DMU—decision-making unit, VRS—Variable Returns to Scale, AUT—Austria, BEL-Belgium, BGR—Bulgaria, CHE-Switzerland, CYP—Cyprus, CZE—The Czech Republic, DEU—Germany, DNK—Denmark, ESP—Spain, EST—Estonia, FIN—Finland, FRA—ZZZ, GBR-United Kingdom, BEL-Belgium, GRC - Greece, HRV—Croatia, HUN-Hungary, IRL—Ireland, ISL-Iceland, ITA—Italy, LTULithuania, LVA—Latvia, MLT—Malta, NLD—Netherlands, NOR—Norway, POL—Poland, PRT—Portugal, ROU—Romania, SVK—Slovak Republic, SVN-Slovenia.

The authors also calculated the efficiency of spending on environmental protection expenditure for an additional ten years (2005-2015). A similar technique was carried out separately for each year. The results of this analysis are presented in Tables 5-7 and in Appendix A from Tables A1-A20.

Table 5. Input-Oriented CRS Efficiency of Environmental Protection Expenditure in 2005-2015. Source: Authors' calculations in DEAFrontier.

\begin{tabular}{|c|c|c|c|c|c|c|c|c|c|c|c|c|c|}
\hline DMU No. & DMU Name & 2015 & 2014 & 2013 & 2012 & 2011 & 2010 & 2009 & 2008 & 2007 & 2006 & 2005 & Avr \\
\hline 1 & FIN & 1.00 & 1.00 & 1.00 & 1.00 & 1.00 & 1.00 & 0.91 & 1.00 & 1.00 & 1.00 & 1.00 & 0.99 \\
\hline 2 & ISL & 0.75 & 1.00 & 1.00 & 0.82 & 1.00 & 0.91 & 0.52 & 1.00 & 1.00 & 0.89 & 0.88 & 0.89 \\
\hline 3 & CYP & 0.65 & 1.00 & 1.00 & 0.85 & 0.92 & 0.83 & 0.51 & 1.00 & 1.00 & 1.00 & 0.86 & 0.87 \\
\hline 4 & AUT & 0.86 & 1.00 & 1.00 & 0.73 & 0.79 & 0.60 & 0.45 & 0.93 & 0.98 & 0.88 & 0.87 & 0.83 \\
\hline 5 & SWE & 0.73 & 1.00 & 0.78 & 0.58 & 0.85 & 0.87 & 0.51 & 0.89 & 0.98 & 0.93 & 0.82 & 0.81 \\
\hline 6 & NOR & 0.49 & 0.74 & 0.62 & 0.60 & 0.73 & 0.65 & 0.38 & 1.00 & 1.00 & 1.00 & 1.00 & 0.75 \\
\hline 7 & LVA & 0.43 & 0.60 & 0.57 & 0.47 & 0.48 & 1.00 & 1.00 & 0.62 & 0.60 & 0.72 & 0.72 & 0.65 \\
\hline 8 & $\mathrm{CHE}$ & 0.47 & 0.69 & 0.62 & 0.49 & 0.55 & 0.52 & 0.29 & 0.90 & 0.83 & 0.64 & 0.58 & 0.60 \\
\hline 9 & HRV & 0.43 & 0.82 & 0.58 & 0.48 & 0.52 & 0.57 & 0.26 & 0.72 & 0.72 & 0.75 & 0.67 & 0.59 \\
\hline 10 & DNK & 0.74 & 0.77 & 0.61 & 0.62 & 0.65 & 0.56 & 0.37 & 0.66 & 0.65 & 0.48 & 0.40 & 0.59 \\
\hline 11 & ROU & 0.30 & 0.50 & 0.39 & 0.36 & 0.36 & 0.37 & 0.32 & 0.82 & 1.00 & 1.00 & 1.00 & 0.58 \\
\hline 12 & PRT & 0.45 & 0.71 & 0.54 & 0.48 & 0.45 & 0.41 & 0.30 & 0.58 & 0.69 & 0.57 & 0.49 & 0.52 \\
\hline 13 & LTU & 0.55 & 0.63 & 0.62 & 0.35 & 0.45 & 0.22 & 0.16 & 0.47 & 0.42 & 0.47 & 0.52 & 0.44 \\
\hline 14 & HUN & 0.23 & 0.28 & 0.25 & 0.40 & 0.44 & 0.47 & 0.31 & 0.60 & 0.58 & 0.49 & 0.47 & 0.41 \\
\hline 15 & DEU & 0.29 & 0.41 & 0.35 & 0.33 & 0.38 & 0.35 & 0.23 & 0.58 & 0.59 & 0.48 & 0.41 & 0.40 \\
\hline 16 & POL & 0.34 & 0.44 & 0.33 & 0.37 & 0.35 & 0.32 & 0.21 & 0.44 & 0.51 & 0.43 & 0.44 & 0.38 \\
\hline 17 & SVN & 0.24 & 0.35 & 0.43 & 0.29 & 0.32 & 0.42 & 0.26 & 0.41 & 0.45 & 0.45 & 0.39 & 0.37 \\
\hline 18 & ITA & 0.29 & 0.40 & 0.33 & 0.28 & 0.30 & 0.31 & 0.20 & 0.42 & 0.47 & 0.46 & 0.32 & 0.34 \\
\hline 19 & EST & 0.20 & 0.30 & 0.30 & 0.16 & 0.72 & 0.75 & 0.25 & 0.22 & 0.27 & 0.35 & 0.27 & 0.34 \\
\hline 20 & BGR & 0.30 & 0.44 & 0.27 & 0.36 & 0.39 & 0.38 & 0.16 & 0.48 & 0.28 & 0.29 & 0.41 & 0.34 \\
\hline
\end{tabular}


Table 5. Cont.

\begin{tabular}{|c|c|c|c|c|c|c|c|c|c|c|c|c|c|}
\hline DMU No. & DMU Name & 2015 & 2014 & 2013 & 2012 & 2011 & 2010 & 2009 & 2008 & 2007 & 2006 & 2005 & Avr \\
\hline 21 & SVK & 0.27 & 0.40 & 0.28 & 0.28 & 0.35 & 0.28 & 0.18 & 0.43 & 0.51 & 0.34 & 0.26 & 0.33 \\
\hline 22 & FRA & 0.28 & 0.33 & 0.24 & 0.27 & 0.30 & 0.27 & 0.18 & 0.41 & 0.40 & 0.39 & 0.31 & 0.31 \\
\hline 23 & IRL & 0.51 & 0.47 & 0.36 & 0.28 & 0.32 & 0.22 & 0.12 & 0.26 & 0.27 & 0.30 & 0.24 & 0.30 \\
\hline 24 & ESP & 0.28 & 0.40 & 0.34 & 0.26 & 0.29 & 0.24 & 0.15 & 0.35 & 0.34 & 0.33 & 0.30 & 0.30 \\
\hline 25 & GBR & 0.30 & 0.36 & 0.25 & 0.29 & 0.30 & 0.23 & 0.15 & 0.33 & 0.32 & 0.31 & 0.17 & 0.27 \\
\hline 26 & GRC & 0.16 & 0.20 & 0.15 & 0.21 & 0.29 & 0.30 & 0.16 & 0.33 & 0.37 & 0.38 & 0.41 & 0.27 \\
\hline 27 & BEL & 0.14 & 0.17 & 0.12 & 0.13 & 0.15 & 0.16 & 0.13 & 0.27 & 0.34 & 0.33 & 0.27 & 0.20 \\
\hline 28 & CZE & 0.15 & 0.21 & 0.19 & 0.14 & 0.16 & 0.19 & 0.18 & 0.28 & 0.25 & 0.23 & 0.20 & 0.20 \\
\hline 29 & MLT & 0.16 & 0.20 & 0.14 & 0.18 & 0.22 & 0.14 & 0.11 & 0.22 & 0.20 & 0.21 & 0.19 & 0.18 \\
\hline 30 & NLD & 0.10 & 0.14 & 0.10 & 0.12 & 0.12 & 0.11 & 0.07 & 0.17 & 0.17 & 0.17 & 0.13 & 0.13 \\
\hline
\end{tabular}

Note: DMU—decision-making unit, AUT-Austria, BEL-Belgium, BGR-Bulgaria, CHE-Switzerland, CYP-Cyprus, CZE-The Czech Republic, DEU—Germany, DNK-Denmark, ESP—Spain, EST—Estonia, FIN_Finland, FRA—ZZZ, GBR-United Kingdom, BELBelgium, GRC - Greece, HRV-Croatia, HUN-Hungary, IRL-Ireland, ISL-Iceland, ITA-Italy, LTU-Lithuania, LVA-Latvia, MLTMalta, NLD—Netherlands, NOR_Norway, POL_Poland, PRT_Portugal, ROU—Romania, SVK-Slovak Republic, SVN-Slovenia.

Table 6. Input-Oriented VRS Efficiency of Environmental Protection Expenditure in 2005-2015. Source: Authors' calculations in DEAFrontier.

\begin{tabular}{|c|c|c|c|c|c|c|c|c|c|c|c|c|c|}
\hline DMU No. & DMU Name & 2015 & 2014 & 2013 & 2012 & 2011 & 2010 & 2009 & 2008 & 2007 & 2006 & 2005 & Average \\
\hline 1 & FIN & 1.00 & 1.00 & 1.00 & 1.00 & 1.00 & 1.00 & 1.00 & 1.00 & 1.00 & 1.00 & 1.00 & 1.00 \\
\hline 2 & ISL & 1.00 & 1.00 & 1.00 & 1.00 & 1.00 & 1.00 & 1.00 & 1.00 & 1.00 & 1.00 & 1.00 & 1.00 \\
\hline 3 & LVA & 1.00 & 1.00 & 1.00 & 1.00 & 1.00 & 1.00 & 1.00 & 1.00 & 1.00 & 1.00 & 1.00 & 1.00 \\
\hline 4 & NOR & 1.00 & 1.00 & 1.00 & 1.00 & 1.00 & 1.00 & 1.00 & 1.00 & 1.00 & 1.00 & 1.00 & 1.00 \\
\hline 5 & SWE & 1.00 & 1.00 & 1.00 & 1.00 & 1.00 & 1.00 & 1.00 & 1.00 & 1.00 & 1.00 & 1.00 & 1.00 \\
\hline 6 & CYP & 0.82 & 1.00 & 1.00 & 1.00 & 1.00 & 1.00 & 0.67 & 1.00 & 1.00 & 1.00 & 1.00 & 0.95 \\
\hline 7 & AUT & 1.00 & 1.00 & 1.00 & 1.00 & 0.85 & 0.69 & 0.63 & 0.98 & 1.00 & 0.89 & 0.87 & 0.90 \\
\hline 8 & ROU & 0.64 & 0.87 & 0.86 & 0.76 & 0.66 & 0.38 & 0.33 & 1.00 & 1.00 & 1.00 & 1.00 & 0.77 \\
\hline 9 & HRV & 0.60 & 1.00 & 0.91 & 0.98 & 0.88 & 0.59 & 0.29 & 0.82 & 0.72 & 0.85 & 0.73 & 0.76 \\
\hline 10 & $\mathrm{CHE}$ & 0.64 & 0.74 & 0.77 & 0.88 & 0.87 & 0.52 & 0.33 & 1.00 & 0.96 & 0.69 & 0.62 & 0.73 \\
\hline 11 & DNK & 0.94 & 0.79 & 0.62 & 0.94 & 0.80 & 0.75 & 0.50 & 0.68 & 0.67 & 0.52 & 0.50 & 0.70 \\
\hline 12 & PRT & 0.59 & 0.79 & 0.81 & 0.94 & 0.79 & 0.43 & 0.33 & 0.68 & 0.70 & 0.58 & 0.50 & 0.65 \\
\hline 13 & LTU & 0.74 & 0.72 & 0.96 & 0.65 & 0.78 & 0.23 & 0.17 & 0.65 & 0.55 & 0.66 & 0.69 & 0.62 \\
\hline 14 & HUN & 0.31 & 0.41 & 0.53 & 0.76 & 0.70 & 0.50 & 0.33 & 0.75 & 0.63 & 0.53 & 0.50 & 0.54 \\
\hline 15 & DEU & 0.33 & 0.50 & 0.42 & 0.33 & 0.39 & 0.50 & 0.33 & 0.60 & 0.61 & 0.50 & 0.50 & 0.46 \\
\hline 16 & EST & 0.29 & 0.43 & 0.37 & 0.22 & 1.00 & 1.00 & 0.25 & 0.27 & 0.33 & 0.37 & 0.33 & 0.44 \\
\hline 17 & POL & 0.36 & 0.50 & 0.37 & 0.40 & 0.37 & 0.43 & 0.29 & 0.44 & 0.52 & 0.43 & 0.50 & 0.42 \\
\hline 18 & SVN & 0.29 & 0.35 & 0.50 & 0.40 & 0.39 & 0.43 & 0.27 & 0.45 & 0.49 & 0.50 & 0.44 & 0.41 \\
\hline 19 & ITA & 0.37 & 0.41 & 0.35 & 0.39 & 0.36 & 0.38 & 0.25 & 0.44 & 0.47 & 0.47 & 0.38 & 0.39 \\
\hline 20 & BGR & 0.35 & 0.44 & 0.30 & 0.48 & 0.45 & 0.43 & 0.18 & 0.55 & 0.30 & 0.31 & 0.43 & 0.38 \\
\hline 21 & FRA & 0.37 & 0.39 & 0.27 & 0.46 & 0.46 & 0.30 & 0.20 & 0.49 & 0.42 & 0.41 & 0.33 & 0.37 \\
\hline 22 & SVK & 0.34 & 0.41 & 0.30 & 0.39 & 0.41 & 0.33 & 0.22 & 0.47 & 0.53 & 0.36 & 0.27 & 0.37 \\
\hline 23 & IRL & 0.52 & 0.50 & 0.40 & 0.31 & 0.35 & 0.30 & 0.18 & 0.27 & 0.30 & 0.33 & 0.33 & 0.34 \\
\hline 24 & ESP & 0.36 & 0.40 & 0.40 & 0.38 & 0.37 & 0.27 & 0.18 & 0.37 & 0.34 & 0.34 & 0.33 & 0.34 \\
\hline 25 & GBR & 0.35 & 0.38 & 0.28 & 0.32 & 0.32 & 0.30 & 0.20 & 0.33 & 0.33 & 0.33 & 0.21 & 0.31 \\
\hline 26 & GRC & 0.19 & 0.21 & 0.15 & 0.24 & 0.32 & 0.38 & 0.22 & 0.33 & 0.38 & 0.39 & 0.50 & 0.30 \\
\hline 27 & CZE & 0.18 & 0.27 & 0.23 & 0.15 & 0.16 & 0.30 & 0.29 & 0.33 & 0.30 & 0.27 & 0.27 & 0.25 \\
\hline 28 & BEL & 0.15 & 0.21 & 0.15 & 0.14 & 0.16 & 0.25 & 0.20 & 0.30 & 0.37 & 0.37 & 0.38 & 0.24 \\
\hline 29 & MLT & 0.32 & 0.20 & 0.14 & 0.21 & 0.26 & 0.16 & 0.13 & 0.27 & 0.21 & 0.23 & 0.21 & 0.21 \\
\hline 30 & NLD & 0.14 & 0.21 & 0.14 & 0.13 & 0.13 & 0.19 & 0.12 & 0.20 & 0.20 & 0.20 & 0.20 & 0.17 \\
\hline
\end{tabular}

Note: DMU—decision-making unit, AUT—Austria, BEL-Belgium, BGR-Bulgaria, CHE-Switzerland, CYP—Cyprus, CZE-The Czech Republic, DEU—Germany, DNK—Denmark, ESP-Spain, EST—Estonia, FIN-Finland, FRA-ZZZ, GBR—United Kingdom, BELBelgium, GRC - Greece, HRV-Croatia, HUN—Hungary, IRL-Ireland, ISL-Iceland, ITA-Italy, LTU—Lithuania, LVA-Latvia, MLTMalta, NLD—Netherlands, NOR—Norway, POL—Poland, PRT—Portugal, ROU—Romania, SVK—Slovak Republic, SVN—Slovenia. 
Table 7. Efficiency Ranking for European Countries. Source: Authors' calculations in DEAFrontier; European Commission [130].

\begin{tabular}{|c|c|c|c|c|c|c|c|}
\hline \multirow{2}{*}{ DMU Name } & CRS & VRS & CRS & VRS & AVG Effectiveness & \multirow{2}{*}{ Rank } & \multirow{2}{*}{$\begin{array}{c}\text { EU Funds } \\
\text { Absorption (\%) }\end{array}$} \\
\hline & Average & Average & Rank & Rank & Index & & \\
\hline FIN & 0.991 & 1.000 & 1 & 1 & 1.00 & 1 & 94.98 \\
\hline ISL & 0.888 & 1.000 & 2 & 1 & 0.94 & 2 & - \\
\hline CYP & 0.874 & 0.953 & 3 & 6 & 0.91 & 3 & 91.93 \\
\hline SWE & 0.813 & 1.000 & 5 & 1 & 0.91 & 4 & 94.71 \\
\hline NOR & 0.747 & 1.000 & 6 & 1 & 0.87 & 5 & - \\
\hline AUT & 0.827 & 0.901 & 4 & 7 & 0.86 & 6 & 89.08 \\
\hline LVA & 0.654 & 1.000 & 7 & 1 & 0.83 & 7 & 95.00 \\
\hline ROU & 0.583 & 0.772 & 11 & 8 & 0.68 & 8 & 70.87 \\
\hline HRV & 0.592 & 0.761 & 9 & 9 & 0.68 & 9 & 58.57 \\
\hline $\mathrm{CHE}$ & 0.597 & 0.730 & 8 & 10 & 0.66 & 10 & - \\
\hline DNK & 0.592 & 0.701 & 10 & 11 & 0.65 & 11 & 95.00 \\
\hline PRT & 0.516 & 0.648 & 12 & 12 & 0.58 & 12 & 94.98 \\
\hline LTU & 0.442 & 0.619 & 13 & 13 & 0.53 & 13 & 94.93 \\
\hline HUN & 0.410 & 0.541 & 14 & 14 & 0.48 & 14 & 88.37 \\
\hline DEU & 0.400 & 0.456 & 15 & 15 & 0.43 & 15 & 91.80 \\
\hline POL & 0.380 & 0.418 & 16 & 17 & 0.40 & 16 & 94.86 \\
\hline EST & 0.344 & 0.443 & 19 & 16 & 0.39 & 17 & 95.00 \\
\hline SVN & 0.365 & 0.410 & 17 & 18 & 0.39 & 18 & 95.00 \\
\hline ITA & 0.344 & 0.387 & 18 & 19 & 0.37 & 19 & 78.97 \\
\hline BGR & 0.341 & 0.383 & 20 & 20 & 0.36 & 20 & 85.24 \\
\hline SVK & 0.328 & 0.368 & 21 & 22 & 0.35 & 21 & 84.36 \\
\hline FRA & 0.308 & 0.373 & 22 & 21 & 0.34 & 22 & 92.12 \\
\hline IRL & 0.303 & 0.345 & 23 & 23 & 0.32 & 23 & 89.96 \\
\hline ESP & 0.299 & 0.341 & 24 & 24 & 0.32 & 24 & 84.07 \\
\hline GBR & 0.274 & 0.306 & 25 & 25 & 0.29 & 25 & 88.01 \\
\hline GRC & 0.270 & 0.301 & 26 & 26 & 0.29 & 26 & 98.09 \\
\hline CZE & 0.197 & 0.251 & 28 & 27 & 0.22 & 27 & 84.13 \\
\hline BEL & 0.201 & 0.244 & 27 & 28 & 0.22 & 28 & 93.06 \\
\hline MLT & 0.179 & 0.214 & 29 & 29 & 0.20 & 29 & 81.61 \\
\hline NLD & 0.127 & 0.169 & 30 & 30 & 0.15 & 30 & 86.54 \\
\hline
\end{tabular}

Note: DMU—decision-making unit, VRS—Variable Returns to Scale, CRS—Constant Returns to Scale, AVG-Average, AUT—Austria, BEL-Belgium, BGR-Bulgaria, CHE-Switzerland, CYP-Cyprus, CZE-The Czech Republic, DEU—Germany, DNK-Denmark, ESPSpain, EST—Estonia, FIN-Finland, FRA-ZZZ, GBR—United Kingdom, BEL—Belgium, GRC—Greece, HRV-Croatia, HUN—Hungary, IRL—Ireland, ISL—Iceland, ITA—Italy, LTU—Lithuania, LVA—Latvia, MLT—Malta, NLD—Netherlands, NOR—Norway, POL—Poland, PRT—Portugal, ROU—Romania, SVK—Slovak Republic, SVN—Slovenia.

Among the analyzed European economies, only Finland was efficient in the CRS model in 2015. Other countries may improve their efficiency by decreasing their environmental protection expenditures. In 2015, the Netherlands achieved the worst results (0.102), and, to become effective, it should receive the same results with inputs that are lower by $88.8 \%$ (1-0.102). Moreover, DEA methodology point out benchmarks (BDMUs), whose environmental strategies, policies and best practices may be a good pattern for ineffective DMUs, to improve their efficiency results. Finland is a benchmark for all other countries, which requires a closer look at the strategies and programs of environmental protection applied in that country. DEA, also, allows to recognize character of return to scale. In our case, most of the countries have decreasing returns to scale. Constant-optimal returns-toscale (RTS) are seen only in Finland. Belgium, the Czech Republic, the Netherlands, Spain and Germany, which all have an increasing RTS.

Six countries are efficient under the VRS model: Austria, Finland, Iceland, Latvia, Norway and Sweden - mainly Nordic countries. Similarly to the CRS model, the Netherlands obtained the worst result (0.143). In general, more economies are efficient under the VRS than the CRS methodology.

The final efficiency index was calculated as the average of the CRS and VRS efficiency based on data from Tables 5 and 6 . The ranking of the most efficient countries is presented in Table 7. According to the presented ranking, Finland is the most efficient country. 
Finland was an efficient DMU in both the CRS and VRS models for the whole analyzed period. Iceland, Norway, Sweden and Latvia (1.00) are efficiency frontiers in the VRS model. Twelve out of thirty economies gained a score higher than average 0.52 in average innovation efficiency index, while the remaining eighteen economies gained scores lower. The Netherland's low place in the ranking may be surprising as this country spends on environmental protection a relatively higher percentage of GDP than other countries in the Europe on environmental protection. The hypothesis about the non-proportional relation between higher environmental protection expenditures and environmental outputs has not been confirmed. From the interpretation of the results, it is essential to emphasize that the DEA methodology calculates relative efficiency, in the study group of countries, and investigates the degree to which environmental protection funds have been converted into environmental outputs.

Some spikes in CRS and VRS efficiencies were caused by unforeseen events. In 2013, one of Belgium's lime plants operated without oxygen and, as a result, in the following year, the efficiency of spending on environmental protection expenditure in Belgium increased from 0.12 to 0.17 (CRS) and from 0.15 to 0.21 (VRS), which was partly due to the elimination of a problem from a previous year, rather than an actual improvement caused by the efficient spending of available funds.

As can be observed in the case of Bulgaria and Romania, two EU Member States since 2007 , a sudden increase in funds for environmental purposes may result in their inefficient use. Romania spent the available funds efficiently in the years before its accession to the EU (VRS and CRS equal to 1), only to experience a decline in subsequent years to 0.82 in 2008 and 0.32 in 2009 (Table 5). In the case of VRS, the change is noticeable starting from 2009. In 2013, Croatia joined the European Union, and, in the second year after joining, a significant decrease in CRS (2013-0.58; 2014-0.82; 2015-0.43) and VRS (2013-0.91; 2014-1; 2015-0.6) values was observed.

A comparison of the ranking of countries in Table 7 and data on the absorption of EU funds in 2015 provides interesting insights. The absorption rate of the EU Cohesion Policy Funds (European Regional Development Fund, Cohesion Fund and European Social Fund) show the percentage paid to each Member State compared to the total available budget. Countries that have absorbed less than $90 \%$ of the funds available to them are: Ireland (rank 23), Hungary (rank 14), UK (rank 25), Netherlands (rank 30), Bulgaria (rank 20), Slovakia (rank 21), Czech Republic (rank 27), Spain (rank 24), Malta (rank 29) and Italy (rank 19). Two cases in particular are worth mentioning. Romania (place 8 ) has the second-worst absorption rate in the whole EU (less than 71\%; the third-worst belongs to Italy, with almost $79 \%)$. Such a high ranking with such a low absorption rate is caused by taking into account data from before Romania's accession to the EU. At the opposite end of the spectrum is Greece (rank 26), which has the highest absorption rate (over 98\%, 3\% higher than the second-best country). This may indicate that inefficiency can be caused not only by the low absorption of funds but also by the fact that the rush to absorb funds can lead to the insufficient consideration of value for money, poor quality of governance and the selection of projects for funding.

Further study of the impact of accession on the efficiency of environmental protection expenditure by comparing the values of CRS and VRS one and two years after the accession of 10 new countries in 2004 to the values of these measures in 2015 reveals that:

- CRS values in 2015 decreased compared to 2005 in 8 of the 10 countries and for VRS in 6 of the 10 countries,

- $\quad$ CRS values in 2015 decreased compared to 2006 in 9 of 10 countries and for VRS in 7 of 10 countries

The thirteen EU countries that joined the EU in 2004, 2007 and 2013 were ranked below the EU average with respect to GDP per capita. However, environmental protection investment in those countries proved to be less efficient than in the case of higher-income Member States, irrespective of the significance of those expenditures for the improvement of environmental quality, life expectancy and related indicators of socioeconomic develop- 
ment, for instance, in Central and Eastern Europe (CEE). CEE countries are heavily affected by the impacts of climate changes, as well as air and noise pollution, on environmental quality and human health when compared to Western Europe. Countries like Poland and Czech Republic are characterized by a concentration of heavy industries and the coal-based energy sector. Both economic development and enforcement have impeded environmental protection investment in CEE [131]. These observations cause one to wonder whether simply comparing the planned budget with its execution is sufficient to assess the performance of individual countries. Our findings are in line with the environmental Kuznets curve, which suggests that economic development initially leads to deterioration in environmental quality, but, after reaching a certain level of economic growth and development, environmental degradation decreases. As argued by Berg et al. [132], in the case of lower income and developing countries, the so-called marginal efficiency, resulting from an increase in public investment, including environmental protection expenditures, generate relatively smaller output multipliers (lower than 1) due to general capital scarcity and historical inefficiencies. The latter involves, among others, lower institutional capacity, governance quality and infrastructure and supply bottlenecks, as well as corruption [133]. Annual reports published by the European Anti-Fraud Office (OLAF) [134] confirm that the problem of fraud of the EU structural funds is more and more significant, with rising number of investigations conducted every year, mostly addressing CEE countries and Italy. The other problems related to the issue, especially in the CEE region are: the selection of contractors under government procurement, budgetary constraints to satisfy national contribution to the project and a heavy reliance on public funds in environmental spending in general.

\section{Conclusions}

Our analysis confirmed the problem of the deteriorating efficiency of environmental expenditures across the selected EU Member States, caused by an increase in spending, whereas Halkos and Paizanos [27] suggested that the reduction of environmental expenditures in higher-income countries, such as the countries of the EU, led to an improvement in environmental quality and an increase in income. This, in turn, supports the argument that, after reaching a given level of income and economic development, environmental quality improves due to rising social pressure to boost spending in this field; however, an increase in environmental expenditures doesn't have to translate into proportional results in terms of their efficiency from country to country, year after year. In other words, continuous increases in government size provide diminishing returns [6]. Therefore, environmental expenditures have to be confronted with environmental outcome to consider efficiency, not only the level of spending on this sectoral policy [103]. The European Green Deal requires dedicated environmental expenditures to achieve the far-reaching goal of carbon neutrality $[6,75,76]$. The problem of carbon dioxide emissions within the single market of the EU, complies with studies by Panayotou [94], Cole and Elliott [95], Dinda [96] and Tsurumi and Managi [97] with respect to the positive correlation between emissions and the degree of trade liberalization, which, in turn, translates into trade creation and diversion effects. Similar to the case of analysis of the EU countries $[16,42,44,46,47,49-52,56,57,70]$, the environmental Kuznets curve hypothesis can be confirmed at the region-level; however, across the selected Member States, results were diversified. For instance, higher-income EU countries relocated dirty industries to lower-income countries, increasing the income and degree of industrialization without the deterioration of environmental quality, as the aforementioned hypothesis assumed. The diversity of environmental outcomes and the efficiency of expenditures proved to vary among the EU Member States due to the diversified approaches and strategies of environmental protection and pollution reduction, which confirms conclusions by Ercolano and Romano [2], based on studies of selected EU Member States. Our research indicated the lack of convergence of public environmental expenditures across the EU Member States, which complies with results achieved by Ercolano and Romano [2], Apergis et al. [105] and Ferreiro et al. [106,107]. The recognition 
of the positive impact of an increase of shares of renewables in final energy consumption supports the empirical findings by Destek et al. [16] that environmental degradation requires a reorientation from fossil-fuels-based growth policies across the Member States. Therefore, EU policymakers are expected to introduce legislative measures to enhance the production of renewable energy, while discouraging non-renewable sources, including dedicated subsidization and tax schemes.

The European Union, when assessing environmental protection expenditures, should focus on the merits of such investments, with particular emphasis on the expected environmental effects. This would result in reports comparing the ratio of expected to achieved environmental effects. Such data would provide more valuable information and conclusions than a simple comparison of planned budget with its execution. The distribution of European funds should depend more on the efficiency of use of those funds, as opposed to the current situation. Inefficient countries should not be punished, but the period of time dedicated to the use of funds should be extended and divided into smaller sub-periods, to avoid motivating politicians in these countries to absorb funds quickly (inefficiently) to serve propaganda purposes. The re-examination of environmental expenditures with a goal of potentially finding and eliminating those expenditures whose classification in that group is not justified due to negligible or no impact at all on the quality of the environment in terms of its protection and the protection of human health. The limitation of the funds allocated for environmental protection might enhance competition among ideas for environmental expenditures.

Our research has some limitations, and they were discussed in the Section 3.

Author Contributions: Conceptualization, A.B., P.D., S.B., K.S. and S.C.; methodology, P.D.; formal analysis, A.B., P.D., S.B., K.S. and S.C.; investigation, A.B., P.D., S.B., K.S. and S.C.; data curation, P.D. and K.S.; writing-original draft preparation, A.B., P.D., S.B., K.S. and S.C.; writing-review and editing, P.D. and S.B.; visualization, P.D. and S.B.; supervision, A.B. All authors have read and agreed to the published version of the manuscript.

Funding: The project is financed by the Ministry of Science and Higher Education in Poland under the program "Regional Initiative of Excellence" 2019-2022 project number 015/RID/2018/19 total funding amount 10,721,040 PLN.

Institutional Review Board Statement: Not applicable.

Informed Consent Statement: Not applicable.

Data Availability Statement: We used publicly available statistical data provided by Eurostat and World Bank in the article.

Acknowledgments: We would like to thank to the Warsaw-Beijing Forum for the opportunity to participate in the conference "Between China and Europe-Energy for Future Earth" in April 2021, and the conference participants for useful comments and to the anonymous referees for their helpful comments and suggestions, which contributed to the quality of the paper. We would also like to issue thanks for allowing me to intern as a Visiting Scholar at the Minda de Gunzburg Center for European Studies (CES) at Harvard University.

Conflicts of Interest: The authors declare no conflict of interest. 


\section{Appendix A}

Table A1. Input-Oriented CRS Efficiency of EPS in 2014. Source: Authors' calculations in DEAFrontier.

\begin{tabular}{|c|c|c|c|c|c|c|c|c|c|c|}
\hline DMU No & DMU Name & CRS Efficiency & S1 & RTS & Benchmarks & DMU & $\lambda$ & DMU & $\lambda$ & DMU \\
\hline 1 & AUT & 1.00000 & 1.000 & Constant & 1.000 & AUT & & & & \\
\hline 2 & BEL & 0.17364 & 0.730 & Increasing & 0.487 & CYP & 0.242 & SWE & & \\
\hline 3 & BGR & 0.44212 & 1.032 & Decreasing & 0.742 & CYP & 0.290 & FIN & & \\
\hline 4 & $\mathrm{CHE}$ & 0.68649 & 1.014 & Decreasing & 0.032 & ISL & 0.982 & SWE & & \\
\hline 5 & CYP & 1.00000 & 1.000 & Constant & 1.000 & CYP & & & & \\
\hline 6 & CZE & 0.21137 & 0.775 & Increasing & 0.407 & CYP & 0.368 & FIN & & \\
\hline 7 & DEU & 0.40879 & 0.672 & Increasing & 0.234 & CYP & 0.437 & SWE & & \\
\hline 8 & DNK & 0.77316 & 0.907 & Increasing & 0.119 & ISL & 0.787 & SWE & & \\
\hline 9 & ESP & 0.40058 & 0.975 & Increasing & 0.295 & CYP & 0.680 & SWE & & \\
\hline 10 & EST & 0.30104 & 0.702 & Increasing & 0.702 & FIN & & & & \\
\hline 11 & FIN & 1.00000 & 1.000 & Constant & 1.000 & FIN & & & & \\
\hline 12 & FRA & 0.33406 & 1.072 & Decreasing & 0.864 & CYP & 0.083 & FIN & 0.124 & SWE \\
\hline 13 & GBR & 0.36340 & 0.881 & Increasing & 0.616 & CYP & 0.265 & SWE & & \\
\hline 14 & GRC & 0.20405 & 0.899 & Increasing & 0.536 & CYP & 0.363 & SWE & & \\
\hline 15 & HRV & 0.82231 & 1.077 & Decreasing & 0.313 & ISL & 0.763 & SWE & & \\
\hline 16 & HUN & 0.27595 & 1.085 & Decreasing & 1.028 & CYP & 0.057 & SWE & & \\
\hline 17 & IRL & 0.46811 & 0.807 & Increasing & 0.418 & CYP & 0.389 & SWE & & \\
\hline 18 & ISL & 1.00000 & 1.000 & Constant & 1.000 & ISL & & & & \\
\hline 19 & ITA & 0.40045 & 0.951 & Increasing & 0.200 & CYP & 0.751 & SWE & & \\
\hline 20 & LTU & 0.62864 & 1.029 & Decreasing & 0.344 & CYP & 0.685 & SWE & & \\
\hline 21 & LVA & 0.60262 & 1.086 & Decreasing & 0.126 & CYP & 0.960 & SWE & & \\
\hline 22 & MLT & 0.19769 & 0.988 & Increasing & 0.988 & CYP & & & & \\
\hline 23 & NLD & 0.13844 & 0.598 & Increasing & 0.455 & CYP & 0.143 & SWE & & \\
\hline 24 & NOR & 0.74448 & 1.110 & Decreasing & 0.704 & AUT & 0.406 & ISL & & \\
\hline 25 & POL & 0.44305 & 0.886 & Increasing & 0.626 & CYP & 0.261 & FIN & & \\
\hline 26 & PRT & 0.70971 & 1.025 & Decreasing & 0.080 & ISL & 0.945 & SWE & & \\
\hline 27 & $\mathrm{ROU}$ & 0.49965 & 1.102 & Decreasing & 0.410 & CYP & 0.692 & SWE & & \\
\hline 28 & SVK & 0.40421 & 1.022 & Decreasing & 0.619 & CYP & 0.236 & FIN & 0.167 & SWE \\
\hline 29 & SVN & 0.34685 & 1.046 & Decreasing & 0.241 & CYP & 0.474 & FIN & 0.331 & SWE \\
\hline 30 & SWE & 1.00000 & 1.000 & Constant & 1.000 & SWE & & & & \\
\hline
\end{tabular}

Note: DMU—decisio-making unit, CRS—Constant Returns to Scale, RTS-Returns to Scale, AUT-Austria, BEL-Belgium, BGRBulgaria, CHE-Switzerland, CYP-Cyprus, CZE-The Czech Republic, DEU—Germany, DNK—Denmark, ESP-Spain, EST—Estonia, FIN-Finland, FRA-ZZZ, GBR-United Kingdom, BEL-Belgium, GRC—Greece, HRV—Croatia, HUN—Hungary, IRL-Ireland, ISLIceland, ITA—Italy, LTU—Lithuania, LVA—Latvia, MLT—Malta, NLD—Netherlands, NOR—Norway, POL—Poland, PRT—Portugal, ROU—Romania, SVK—Slovak Republic, SVN-Slovenia.

Table A2. Input-Oriented VRS Efficiency of EPS in 2014. Source: Authors' calculations in DEAFrontier.

\begin{tabular}{ccccccccc}
\hline DMU No & DMU Name & VRS Efficiency & Benchmarks & DMU & $\boldsymbol{\lambda}$ & DMU & $\boldsymbol{\lambda}$ & DMU \\
\hline 1 & AUT & 1.00000 & 1.000 & AUT & & & & \\
2 & BEL & 0.21429 & 0.687 & CYP & 0.313 & FIN & \\
3 & BGR & 0.44393 & 0.691 & CYP & 0.201 & FIN & 0.108 & SWE \\
4 & CHE & 0.74066 & 0.125 & HRV & 0.065 & LVA & 0.810 & SWE \\
5 & CYP & 1.00000 & 1.000 & CYP & & & & \\
\hline
\end{tabular}


Table A2. Cont.

\begin{tabular}{|c|c|c|c|c|c|c|c|c|}
\hline DMU No & DMU Name & VRS Efficiency & Benchmarks & DMU & $\lambda$ & DMU & $\lambda$ & DMU \\
\hline 6 & CZE & 0.27273 & 0.709 & CYP & 0.291 & FIN & & \\
\hline 7 & DEU & 0.50000 & 0.398 & CYP & 0.602 & FIN & & \\
\hline 8 & DNK & 0.78547 & 0.165 & AUT & 0.238 & FIN & 0.597 & SWE \\
\hline 9 & ESP & 0.40295 & 0.297 & CYP & 0.077 & FIN & 0.627 & SWE \\
\hline 10 & EST & 0.42857 & 1.000 & FIN & & & & \\
\hline 11 & FIN & 1.00000 & 1.000 & FIN & & & & \\
\hline 12 & FRA & 0.38855 & 0.115 & CYP & 0.885 & SWE & & \\
\hline 13 & GBR & 0.37623 & 0.623 & CYP & 0.367 & FIN & 0.010 & SWE \\
\hline 14 & GRC & 0.20984 & 0.541 & CYP & 0.311 & FIN & 0.148 & SWE \\
\hline 15 & HRV & 1.00000 & 1.000 & HRV & & & & \\
\hline 16 & HUN & 0.40620 & 0.291 & LVA & 0.709 & SWE & & \\
\hline 17 & IRL & 0.50000 & 0.442 & CYP & 0.558 & FIN & & \\
\hline 18 & ISL & 1.00000 & 1.000 & ISL & & & & \\
\hline 19 & ITA & 0.40514 & 0.203 & CYP & 0.151 & FIN & 0.646 & SWE \\
\hline 20 & LTU & 0.71938 & 0.105 & LVA & 0.895 & SWE & & \\
\hline 21 & LVA & 1.00000 & 1.000 & LVA & & & & \\
\hline 22 & MLT & 0.20000 & 1.000 & CYP & & & & \\
\hline 23 & NLD & 0.21429 & 0.871 & CYP & 0.129 & FIN & & \\
\hline 24 & NOR & 1.00000 & 1.000 & NOR & & & & \\
\hline 25 & POL & 0.50000 & 0.779 & CYP & 0.221 & FIN & & \\
\hline 26 & PRT & 0.78536 & 0.274 & HRV & 0.054 & LVA & 0.671 & SWE \\
\hline 27 & ROU & 0.86903 & 0.984 & LVA & 0.016 & SWE & & \\
\hline 28 & SVK & 0.40532 & 0.583 & CYP & 0.174 & FIN & 0.243 & SWE \\
\hline 29 & SVN & 0.34868 & 0.168 & CYP & 0.346 & FIN & 0.487 & SWE \\
\hline 30 & SWE & 1.00000 & 1.000 & SWE & & & & \\
\hline
\end{tabular}

Note: DMU—decision-making unit, VRS—Variable Returns to Scale, AUT-Austria, BEL-Belgium, BGR-Bulgaria, CHE-Switzerland, CYP-Cyprus, CZE-The Czech Republic, DEU-Germany, DNK-Denmark, ESP-Spain, EST-Estonia, FIN-Finland, FRA-ZZZ, GBR-United Kingdom, BEL-Belgium, GRC_-Greece, HRV-Croatia, HUN-Hungary, IRL-Ireland, ISL-Iceland, ITA-Italy, LTULithuania, LVA—Latvia, MLT—Malta, NLD—Netherlands, NOR—Norway, POL—Poland, PRT—Portugal, ROU—Romania, SVK—Slovak Republic, SVN-Slovenia.

Table A3. Input-Oriented CRS Efficiency of EPS in 2013. Source: Authors' calculations in DEAFrontier.

\begin{tabular}{|c|c|c|c|c|c|c|c|c|c|c|}
\hline DMU No & DMU Name & CRS Efficiency & S1 & RTS & Benchmarks & DMU & $\lambda$ & DMU & $\lambda$ & DMU \\
\hline 1 & AUT & 1.00000 & 1.000 & Constant & 1.000 & AUT & & & & \\
\hline 2 & BEL & 0.12063 & 0.776 & Increasing & 0.072 & AUT & 0.589 & CYP & 0.115 & FIN \\
\hline 3 & BGR & 0.26565 & 1.054 & Decreasing & 0.002 & AUT & 0.774 & CYP & 0.278 & FIN \\
\hline 4 & $\mathrm{CHE}$ & 0.61691 & 1.133 & Decreasing & 0.718 & AUT & 0.414 & CYP & & \\
\hline 5 & CYP & 1.00000 & 1.000 & Constant & 1.000 & CYP & & & & \\
\hline 6 & CZE & 0.18907 & 0.759 & Increasing & 0.386 & CYP & 0.373 & FIN & & \\
\hline 7 & DEU & 0.35291 & 0.776 & Increasing & 0.156 & AUT & 0.366 & CYP & 0.254 & FIN \\
\hline 8 & DNK & 0.61121 & 0.981 & Increasing & 0.547 & AUT & 0.435 & CYP & & \\
\hline 9 & ESP & 0.34435 & 1.098 & Decreasing & 0.409 & AUT & 0.603 & CYP & 0.085 & FIN \\
\hline 10 & EST & 0.30109 & 0.703 & Increasing & 0.703 & FIN & & & & \\
\hline 11 & FIN & 1.00000 & 1.000 & Constant & 1.000 & FIN & & & & \\
\hline 12 & FRA & 0.24134 & 1.064 & Decreasing & 0.060 & AUT & 0.837 & CYP & 0.166 & FIN \\
\hline 13 & GBR & 0.24844 & 0.876 & Increasing & 0.118 & AUT & 0.758 & CYP & & \\
\hline 14 & GRC & 0.14637 & 0.981 & Increasing & 0.198 & AUT & 0.653 & CYP & 0.130 & FIN \\
\hline 15 & HRV & 0.57892 & 1.213 & Decreasing & 0.813 & AUT & 0.400 & CYP & & \\
\hline 16 & HUN & 0.24552 & 1.082 & Decreasing & 1.035 & CYP & 0.047 & FIN & & \\
\hline 17 & IRL & 0.35975 & 0.860 & Increasing & 0.219 & AUT & 0.640 & CYP & & \\
\hline 18 & ISL & 1.00000 & 1.000 & Constant & 1.000 & ISL & & & & \\
\hline 19 & ITA & 0.32730 & 1.032 & Decreasing & 0.441 & AUT & 0.591 & CYP & & \\
\hline 20 & LTU & 0.61595 & 1.144 & Decreasing & 0.369 & AUT & 0.721 & CYP & 0.054 & FIN \\
\hline 21 & LVA & 0.56580 & 1.286 & Decreasing & 0.566 & AUT & 0.463 & CYP & 0.257 & FIN \\
\hline 22 & MLT & 0.13953 & 0.977 & Increasing & 0.977 & CYP & & & & \\
\hline 23 & NLD & 0.09767 & 0.631 & Increasing & 0.102 & AUT & 0.529 & CYP & & \\
\hline 24 & NOR & 0.61940 & 1.132 & Decreasing & 0.704 & AUT & 0.428 & ISL & & \\
\hline 25 & POL & 0.33222 & 0.863 & Increasing & 0.596 & CYP & 0.267 & FIN & & \\
\hline
\end{tabular}


Table A3. Cont.

\begin{tabular}{|c|c|c|c|c|c|c|c|c|c|c|}
\hline DMU No & DMU Name & CRS Efficiency & S1 & RTS & Benchmarks & DMU & $\lambda$ & DMU & $\lambda$ & DMU \\
\hline 26 & PRT & 0.53704 & 1.179 & Decreasing & 0.701 & AUT & 0.478 & CYP & & \\
\hline 27 & ROU & 0.38597 & 1.183 & Decreasing & 0.361 & AUT & 0.822 & CYP & & \\
\hline 28 & SVK & 0.28286 & 1.034 & Decreasing & 0.062 & AUT & 0.618 & CYP & 0.354 & FIN \\
\hline 29 & SVN & 0.42832 & 1.085 & Decreasing & 0.042 & AUT & 0.299 & CYP & 0.744 & FIN \\
\hline 30 & SWE & 0.78390 & 1.267 & Decreasing & 0.373 & AUT & 0.256 & CYP & 0.638 & FIN \\
\hline
\end{tabular}

Note: DMU—decision-making unit, CRS-Constant Returns to Scale, RTS-Returns to Scale, AUT-Austria, BEL-Belgium, BGRBulgaria, CHE-Switzerland, CYP_Cyprus, CZE-The Czech Republic, DEU—Germany, DNK-Denmark, ESP-Spain, EST-Estonia, FIN-Finland, FRA_ZZZ, GBR_United Kingdom, BEL_Belgium, GRC_-Greece, HRV-Croatia, HUN-Hungary, IRL-Ireland, ISLIceland, ITA-Italy, LTU_Lithuania, LVA-Latvia, MLT-Malta, NLD—Netherlands, NOR-Norway, POL-Poland, PRT-Portugal, ROU-Romania, SVK-Slovak Republic, SVN-Slovenia.

Table A4. Input-Oriented VRS Efficiency of EPS in 2013. Source: Authors' calculations in DEAFrontier.

\begin{tabular}{|c|c|c|c|c|c|c|c|c|c|c|}
\hline DMU No & DMU Name & VRS Efficiency & Benchmarks & DMU & $\lambda$ & DMU & $\lambda$ & DMU & $\lambda$ & DMU \\
\hline 1 & AUT & 1.00000 & 1.000 & AUT & & & & & & \\
\hline 2 & BEL & 0.14624 & 0.080 & AUT & 0.886 & CYP & 0.033 & FIN & & \\
\hline 3 & BGR & 0.30371 & 0.686 & CYP & 0.104 & FIN & 0.210 & SWE & & \\
\hline 4 & $\mathrm{CHE}$ & 0.77437 & 0.233 & CYP & 0.337 & ISL & 0.173 & LVA & 0.257 & SWE \\
\hline 5 & CYP & 1.00000 & 1.000 & CYP & & & & & & \\
\hline 6 & $\mathrm{CZE}$ & 0.22902 & 0.710 & CYP & 0.290 & FIN & & & & \\
\hline 7 & DEU & 0.41683 & 0.164 & AUT & 0.663 & CYP & 0.173 & FIN & & \\
\hline 8 & DNK & 0.61782 & 0.545 & AUT & 0.455 & CYP & & & & \\
\hline 9 & ESP & 0.40496 & 0.003 & AUT & 0.451 & CYP & 0.139 & ISL & 0.407 & SWE \\
\hline 10 & EST & 0.37148 & 0.400 & CYP & 0.600 & FIN & & & & \\
\hline 11 & FIN & 1.00000 & 1.000 & FIN & & & & & & \\
\hline 12 & FRA & 0.27389 & 0.760 & CYP & 0.009 & LVA & 0.231 & SWE & & \\
\hline 13 & GBR & 0.27616 & 0.105 & AUT & 0.895 & CYP & & & & \\
\hline 14 & GRC & 0.14830 & 0.199 & AUT & 0.678 & CYP & 0.123 & FIN & & \\
\hline 15 & HRV & 0.91378 & 0.022 & CYP & 0.247 & ISL & 0.731 & LVA & & \\
\hline 16 & HUN & 0.52732 & 0.451 & CYP & 0.549 & LVA & & & & \\
\hline 17 & IRL & 0.40143 & 0.204 & AUT & 0.796 & CYP & & & & \\
\hline 18 & ISL & 1.00000 & 1.000 & ISL & & & & & & \\
\hline 19 & ITA & 0.34942 & 0.254 & AUT & 0.534 & CYP & 0.077 & ISL & 0.135 & SWE \\
\hline 20 & LTU & 0.96359 & 0.431 & CYP & 0.013 & ISL & 0.556 & LVA & & \\
\hline 21 & LVA & 1.00000 & 1.000 & LVA & & & & & & \\
\hline 22 & MLT & 0.14286 & 1.000 & CYP & & & & & & \\
\hline 23 & NLD & 0.14158 & 0.062 & AUT & 0.938 & CYP & & & & \\
\hline 24 & NOR & 1.00000 & 1.000 & NOR & & & & & & \\
\hline 25 & POL & 0.37002 & 0.780 & CYP & 0.220 & FIN & & & & \\
\hline 26 & PRT & 0.81216 & 0.171 & CYP & 0.229 & ISL & 0.600 & LVA & & \\
\hline 27 & ROU & 0.86313 & 0.019 & CYP & 0.981 & LVA & & & & \\
\hline 28 & SVK & 0.29808 & 0.015 & AUT & 0.585 & CYP & 0.273 & FIN & 0.127 & SWE \\
\hline 29 & SVN & 0.49593 & 0.180 & CYP & 0.494 & FIN & 0.326 & SWE & & \\
\hline 30 & SWE & 1.00000 & 1.000 & SWE & & & & & & \\
\hline
\end{tabular}

Note: DMU—decision-making unit, VRS—Variable Returns to Scale, AUT_-Austria, BEL-Belgium, BGR-Bulgaria, CHE-Switzerland, CYP-Cyprus, CZE-The Czech Republic, DEU—Germany, DNK-Denmark, ESP-Spain, EST-Estonia, FIN-Finland, FRA-ZZZ, GBR-United Kingdom, BEL-Belgium, GRC_-Greece, HRV-Croatia, HUN-Hungary, IRL-Ireland, ISL-Iceland, ITA-Italy, LTULithuania, LVA-Latvia, MLT-Malta, NLD—Netherlands, NOR-Norway, POL_Poland, PRT-Portugal, ROU-Romania, SVK-Slovak Republic, SVN-Slovenia.

Table A5. Input-Oriented VRS Efficiency of EPS in 2012. Source: Authors' calculations in DEAFrontier.

\begin{tabular}{|c|c|c|c|c|c|c|}
\hline DMU No & DMU Name & CRS Efficiency & S1 & RTS & Benchmarks & DMU \\
\hline 1 & AUT & 0.73497 & 1.837 & Decreasing & 1.837 & FIN \\
\hline 2 & BEL & 0.13060 & 1.045 & Decreasing & 1.045 & FIN \\
\hline 3 & BGR & 0.36206 & 1.267 & Decreasing & 1.267 & FIN \\
\hline 4 & $\mathrm{CHE}$ & 0.48876 & 1.466 & Decreasing & 1.466 & FIN \\
\hline 5 & CYP & 0.84751 & 1.271 & Decreasing & 1.271 & FIN \\
\hline
\end{tabular}


Table A5. Cont.

\begin{tabular}{|c|c|c|c|c|c|c|}
\hline DMU No & DMU Name & CRS Efficiency & S1 & RTS & Benchmarks & DMU \\
\hline 6 & CZE & 0.14284 & 0.928 & Increasing & 0.928 & FIN \\
\hline 7 & DEU & 0.32952 & 0.989 & Increasing & 0.989 & FIN \\
\hline 8 & DNK & 0.61644 & 1.233 & Decreasing & 1.233 & FIN \\
\hline 9 & ESP & 0.26224 & 1.311 & Decreasing & 1.311 & FIN \\
\hline 10 & EST & 0.16013 & 0.721 & Increasing & 0.721 & FIN \\
\hline 11 & FIN & 1.00000 & 1.000 & Constant & 1.000 & FIN \\
\hline 12 & FRA & 0.27299 & 1.365 & Decreasing & 1.365 & FIN \\
\hline 13 & GBR & 0.28904 & 1.156 & Decreasing & 1.156 & FIN \\
\hline 14 & GRC & 0.21193 & 1.166 & Decreasing & 1.166 & FIN \\
\hline 15 & HRV & 0.48088 & 1.443 & Decreasing & 1.443 & FIN \\
\hline 16 & HUN & 0.40357 & 1.412 & Decreasing & 1.412 & FIN \\
\hline 17 & IRL & 0.28018 & 1.121 & Decreasing & 1.121 & FIN \\
\hline 18 & ISL & 0.82165 & 2.465 & Decreasing & 2.465 & FIN \\
\hline 19 & ITA & 0.28028 & 1.261 & Decreasing & 1.261 & FIN \\
\hline 20 & LTU & 0.35146 & 1.406 & Decreasing & 1.406 & FIN \\
\hline 21 & LVA & 0.46933 & 1.643 & Decreasing & 1.643 & FIN \\
\hline 22 & MLT & 0.17787 & 1.245 & Decreasing & 1.245 & FIN \\
\hline 23 & NLD & 0.11867 & 0.890 & Increasing & 0.890 & FIN \\
\hline 24 & NOR & 0.60374 & 2.415 & Decreasing & 2.415 & FIN \\
\hline 25 & POL & 0.36922 & 1.108 & Decreasing & 1.108 & FIN \\
\hline 26 & PRT & 0.47636 & 1.429 & Decreasing & 1.429 & FIN \\
\hline 27 & ROU & 0.36419 & 1.457 & Decreasing & 1.457 & FIN \\
\hline 28 & SVK & 0.28311 & 1.274 & Decreasing & 1.274 & FIN \\
\hline 29 & SVN & 0.29402 & 1.176 & Decreasing & 1.176 & FIN \\
\hline 30 & SWE & 0.58250 & 1.456 & Decreasing & 1.456 & FIN \\
\hline
\end{tabular}

Note: DMU—decision-making unit, CRS-Constant Returns to Scale, RTS-Returns to Scale, AUT-Austria, BEL-Belgium, BGRBulgaria, CHE—Switzerland, CYP-Cyprus, CZE—The Czech Republic, DEU—Germany, DNK-Denmark, ESP-Spain, EST-Estonia, FIN-Finland, FRA-ZZZ, GBR_United Kingdom, BEL_Belgium, GRC_-Greece, HRV-Croatia, HUN-Hungary, IRL-Ireland, ISLIceland, ITA-Italy, LTU—Lithuania, LVA—Latvia, MLT—Malta, NLD—Netherlands, NOR—Norway, POL-Poland, PRT—Portugal, ROU-Romania, SVK—Slovak Republic, SVN-Slovenia.

Table A6. Input-Oriented VRS Efficiency of EPS in 2012. Source: Authors' calculations in DEAFrontier.

\begin{tabular}{|c|c|c|c|c|c|c|c|c|}
\hline DMU No. & DMU Name & VRS Efficiency & Benchmarks & DMU & $\lambda$ & DMU & $\lambda$ & DMU \\
\hline 1 & AUT & 1.00000 & 1.000 & AUT & & & & \\
\hline 2 & BEL & 0.13531 & 0.165 & CYP & 0.835 & FIN & & \\
\hline 3 & BGR & 0.47544 & 0.689 & CYP & 0.098 & FIN & 0.213 & SWE \\
\hline 4 & $\mathrm{CHE}$ & 0.87962 & 0.014 & CYP & 0.153 & LVA & 0.833 & SWE \\
\hline 5 & CYP & 1.00000 & 1.000 & CYP & & & & \\
\hline 6 & CZE & 0.15385 & 1.000 & FIN & & & & \\
\hline 7 & DEU & 0.33333 & 1.000 & FIN & & & & \\
\hline 8 & DNK & 0.94122 & 0.076 & CYP & 0.361 & FIN & 0.563 & SWE \\
\hline 9 & ESP & 0.38480 & 0.542 & CYP & 0.023 & FIN & 0.435 & SWE \\
\hline 10 & EST & 0.22222 & 1.000 & FIN & & & & \\
\hline 11 & FIN & 1.00000 & 1.000 & FIN & & & & \\
\hline 12 & FRA & 0.45565 & 0.611 & CYP & 0.389 & LVA & & \\
\hline 13 & GBR & 0.32196 & 0.576 & CYP & 0.424 & FIN & & \\
\hline 14 & GRC & 0.23733 & 0.611 & CYP & 0.389 & FIN & & \\
\hline 15 & HRV & 0.97548 & 0.274 & CYP & 0.700 & LVA & 0.026 & SWE \\
\hline 16 & HUN & 0.76372 & 0.413 & CYP & 0.587 & LVA & & \\
\hline 17 & IRL & 0.30562 & 0.445 & CYP & 0.555 & FIN & & \\
\hline 18 & ISL & 1.00000 & 1.000 & ISL & & & & \\
\hline 19 & ITA & 0.39373 & 0.462 & CYP & 0.178 & FIN & 0.361 & SWE \\
\hline 20 & LTU & 0.65444 & 0.441 & CYP & 0.559 & LVA & & \\
\hline 21 & LVA & 1.00000 & 1.000 & LVA & & & & \\
\hline 22 & MLT & 0.20739 & 0.903 & CYP & 0.097 & FIN & & \\
\hline 23 & NLD & 0.13333 & 1.000 & FIN & & & & \\
\hline 24 & NOR & 1.00000 & 1.000 & NOR & & & & \\
\hline
\end{tabular}


Table A6. Cont.

\begin{tabular}{|c|c|c|c|c|c|c|c|c|}
\hline DMU No. & DMU Name & VRS Efficiency & Benchmarks & DMU & $\lambda$ & DMU & $\lambda$ & DMU \\
\hline 25 & POL & 0.39948 & 0.397 & CYP & 0.603 & FIN & & \\
\hline 26 & PRT & 0.93697 & 0.345 & CYP & 0.655 & LVA & & \\
\hline 27 & ROU & 0.76021 & 0.230 & CYP & 0.770 & LVA & & \\
\hline 28 & SVK & 0.38997 & 0.579 & CYP & 0.111 & FIN & 0.310 & SWE \\
\hline 29 & SVN & 0.39908 & 0.180 & CYP & 0.483 & FIN & 0.338 & SWE \\
\hline 30 & SWE & 1.00000 & 1.000 & SWE & & & & \\
\hline
\end{tabular}

Note: DMU—decision-making unit, VRS—Variable Returns to Scale, AUT—Austria, BEL—Belgium, BGR—Bulgaria, CHE-Switzerland, CYP-Cyprus, CZE-The Czech Republic, DEU—Germany, DNK—Denmark, ESP—Spain, EST—Estonia, FIN—Finland, FRA—ZZZ, GBR-United Kingdom, BEL—Belgium, GRC - Greece, HRV-Croatia, HUN—Hungary, IRL-Ireland, ISL-Iceland, ITA-Italy, LTU— Lithuania, LVA—Latvia, MLT—Malta, NLD—Netherlands, NOR—Norway, POL—Poland, PRT—Portugal, ROU—Romania, SVK—Slovak Republic, SVN-Slovenia.

Table A7. Input-Oriented CRS Efficiency of EPS in 2011. Source: Authors' calculations in DEAFrontier.

\begin{tabular}{|c|c|c|c|c|c|c|c|c|}
\hline DMU No & DMU Name & CRS Efficiency & S1 & RTS & Benchmarks & DMU & $\lambda$ & DMU \\
\hline 1 & AUT & 0.79102 & 1.084 & Decreasing & 0.637 & FIN & 0.447 & ISL \\
\hline 2 & BEL & 0.15186 & 1.139 & Decreasing & 1.139 & FIN & & \\
\hline 3 & BGR & 0.38908 & 1.362 & Decreasing & 1.362 & FIN & & \\
\hline 4 & $\mathrm{CHE}$ & 0.54745 & 1.529 & Decreasing & 1.472 & FIN & 0.057 & ISL \\
\hline 5 & CYP & 0.91514 & 1.373 & Decreasing & 1.373 & FIN & & \\
\hline 6 & $\mathrm{CZE}$ & 0.15717 & 1.022 & Decreasing & 1.022 & FIN & & \\
\hline 7 & DEU & 0.37855 & 1.136 & Decreasing & 1.136 & FIN & & \\
\hline 8 & DNK & 0.65318 & 1.306 & Decreasing & 1.306 & FIN & & \\
\hline 9 & ESP & 0.29004 & 1.450 & Decreasing & 1.450 & FIN & & \\
\hline 10 & EST & 0.72070 & 0.721 & Increasing & 0.721 & FIN & & \\
\hline 11 & FIN & 1.00000 & 1.000 & Constant & 1.000 & FIN & & \\
\hline 12 & FRA & 0.30356 & 1.518 & Decreasing & 1.518 & FIN & & \\
\hline 13 & GBR & 0.29507 & 1.328 & Decreasing & 1.328 & FIN & & \\
\hline 14 & GRC & 0.29260 & 1.317 & Decreasing & 1.317 & FIN & & \\
\hline 15 & HRV & 0.51943 & 1.558 & Decreasing & 1.558 & FIN & & \\
\hline 16 & HUN & 0.43924 & 1.537 & Decreasing & 1.537 & FIN & & \\
\hline 17 & IRL & 0.31511 & 1.260 & Decreasing & 1.260 & FIN & & \\
\hline 18 & ISL & 1.00000 & 1.000 & Constant & 1.000 & ISL & & \\
\hline 19 & ITA & 0.30291 & 1.363 & Decreasing & 1.363 & FIN & & \\
\hline 20 & LTU & 0.44793 & 1.568 & Decreasing & 1.568 & FIN & & \\
\hline 21 & LVA & 0.47546 & 1.664 & Decreasing & 1.664 & FIN & & \\
\hline 22 & MLT & 0.21821 & 1.418 & Decreasing & 1.418 & FIN & & \\
\hline 23 & NLD & 0.12488 & 0.999 & Increasing & 0.999 & FIN & & \\
\hline 24 & NOR & 0.72527 & 1.266 & Decreasing & 0.448 & FIN & 0.818 & ISL \\
\hline 25 & POL & 0.34520 & 1.208 & Decreasing & 1.208 & FIN & & \\
\hline 26 & PRT & 0.44908 & 1.572 & Decreasing & 1.572 & FIN & & \\
\hline 27 & $\mathrm{ROU}$ & 0.35518 & 1.598 & Decreasing & 1.598 & FIN & & \\
\hline 28 & SVK & 0.34809 & 1.392 & Decreasing & 1.392 & FIN & & \\
\hline 29 & SVN & 0.32454 & 1.298 & Decreasing & 1.298 & FIN & & \\
\hline 30 & SWE & 0.84770 & 1.419 & Decreasing & 1.281 & FIN & 0.138 & ISL \\
\hline
\end{tabular}

Note: DMU—decision-making unit, CRS—Constant Returns to Scale, RTS-Returns to Scale, AUT-Austria, BEL-Belgium, BGRBulgaria, CHE-Switzerland, CYP—Cyprus, CZE—The Czech Republic, DEU—Germany, DNK—Denmark, ESP-Spain, EST—Estonia, FIN-Finland, FRA-ZZZ, GBR-United Kingdom, BEL-Belgium, GRC-Greece, HRV-Croatia, HUN-Hungary, IRL-Ireland, ISLIceland, ITA—Italy, LTU—Lithuania, LVA—Latvia, MLT—Malta, NLD—Netherlands, NOR—Norway, POL—Poland, PRT—Portugal, ROU—Romania, SVK-Slovak Republic, SVN-Slovenia. 
Table A8. Input-Oriented VRS Efficiency of EPS in 2011. Source: Authors' calculations in DEAFrontier.

\begin{tabular}{|c|c|c|c|c|c|c|c|c|}
\hline DMU No. & DMU Name & VRS Efficiency & Benchmarks & DMU & $\lambda$ & DMU & $\lambda$ & DMU \\
\hline 1 & AUT & 0.84669 & 0.217 & FIN & 0.333 & ISL & 0.450 & SWE \\
\hline 2 & BEL & 0.15819 & 0.373 & CYP & 0.627 & FIN & & \\
\hline 3 & BGR & 0.44661 & 0.689 & CYP & 0.092 & FIN & 0.218 & SWE \\
\hline 4 & $\mathrm{CHE}$ & 0.87065 & 0.008 & ISL & 0.403 & LVA & 0.589 & SWE \\
\hline 5 & CYP & 1.00000 & 1.000 & CYP & & & & \\
\hline 6 & CZE & 0.15831 & 0.058 & CYP & 0.942 & FIN & & \\
\hline 7 & DEU & 0.39399 & 0.364 & CYP & 0.636 & FIN & & \\
\hline 8 & DNK & 0.79760 & 0.156 & CYP & 0.327 & FIN & 0.517 & SWE \\
\hline 9 & ESP & 0.37221 & 0.278 & CYP & 0.722 & SWE & & \\
\hline 10 & EST & 1.00000 & 1.000 & EST & & & & \\
\hline 11 & FIN & 1.00000 & 1.000 & FIN & & & & \\
\hline 12 & FRA & 0.46159 & 0.205 & LVA & 0.795 & SWE & & \\
\hline 13 & GBR & 0.32301 & 0.830 & CYP & 0.132 & FIN & 0.039 & SWE \\
\hline 14 & GRC & 0.32413 & 0.728 & CYP & 0.178 & FIN & 0.095 & SWE \\
\hline 15 & HRV & 0.87932 & 0.425 & LVA & 0.575 & SWE & & \\
\hline 16 & HUN & 0.70493 & 0.312 & LVA & 0.688 & SWE & & \\
\hline 17 & IRL & 0.34839 & 0.539 & CYP & 0.337 & FIN & 0.124 & SWE \\
\hline 18 & ISL & 1.00000 & 1.000 & ISL & & & & \\
\hline 19 & ITA & 0.36072 & 0.482 & CYP & 0.136 & FIN & 0.382 & SWE \\
\hline 20 & LTU & 0.77568 & 0.477 & LVA & 0.523 & SWE & & \\
\hline 21 & LVA & 1.00000 & 1.000 & LVA & & & & \\
\hline 22 & MLT & 0.26349 & 0.575 & CYP & 0.425 & SWE & & \\
\hline 23 & NLD & 0.12500 & 0.003 & EST & 0.997 & FIN & & \\
\hline 24 & NOR & 1.00000 & 1.000 & NOR & & & & \\
\hline 25 & POL & 0.36552 & 0.559 & CYP & 0.441 & FIN & & \\
\hline 26 & PRT & 0.78510 & 0.499 & LVA & 0.501 & SWE & & \\
\hline 27 & ROU & 0.65865 & 0.643 & LVA & 0.357 & SWE & & \\
\hline 28 & SVK & 0.41493 & 0.570 & CYP & 0.055 & FIN & 0.375 & SWE \\
\hline 29 & SVN & 0.39401 & 0.163 & CYP & 0.342 & FIN & 0.494 & SWE \\
\hline 30 & SWE & 1.00000 & 1.000 & SWE & & & & \\
\hline
\end{tabular}

Note: DMU—decision-making unit, VRS—Variable Returns to Scale, AUT-Austria, BEL-Belgium, BGR—Bulgaria, CHE-Switzerland, CYP-Cyprus, CZE-The Czech Republic, DEU—Germany, DNK-Denmark, ESP-Spain, EST-Estonia, FIN-Finland, FRA-ZZZ, GBR-United Kingdom, BEL—Belgium, GRC - Greece, HRV—Croatia, HUN—Hungary, IRL-Ireland, ISL-Iceland, ITA-Italy, LTU— Lithuania, LVA—Latvia, MLT-Malta, NLD—Netherlands, NOR-Norway, POL—Poland, PRT—Portugal, ROU—Romania, SVK-Slovak Republic, SVN-Slovenia.

Table A9. Input-Oriented CRS Efficiency of EPS in 2010. Source: Authors' calculations in DEAFrontier.

\begin{tabular}{|c|c|c|c|c|c|c|c|c|}
\hline DMU No & DMU Name & CRS Efficiency & S1 & RTS & Benchmarks & DMU & $\lambda$ & DMU \\
\hline 1 & AUT & 0.60355 & 1.207 & Decreasing & 1.207 & LVA & & \\
\hline 2 & BEL & 0.16187 & 0.647 & Increasing & 0.647 & LVA & & \\
\hline 3 & BGR & 0.37760 & 0.881 & Increasing & 0.881 & LVA & & \\
\hline 4 & $\mathrm{CHE}$ & 0.51712 & 1.034 & Decreasing & 1.034 & LVA & & \\
\hline 5 & CYP & 0.82692 & 0.827 & Increasing & 0.827 & LVA & & \\
\hline 6 & CZE & 0.18783 & 0.626 & Increasing & 0.034 & FIN & 0.592 & LVA \\
\hline 7 & DEU & 0.34768 & 0.695 & Increasing & 0.695 & LVA & & \\
\hline 8 & DNK & 0.56408 & 0.752 & Increasing & 0.752 & LVA & & \\
\hline 9 & ESP & 0.24257 & 0.889 & Increasing & 0.889 & LVA & & \\
\hline 10 & EST & 0.74646 & 0.746 & Increasing & 0.649 & FIN & 0.097 & LVA \\
\hline 11 & FIN & 1.00000 & 1.000 & Constant & 1.000 & FIN & & \\
\hline 12 & FRA & 0.27336 & 0.911 & Increasing & 0.911 & LVA & & \\
\hline 13 & GBR & 0.23253 & 0.775 & Increasing & 0.775 & LVA & & \\
\hline 14 & GRC & 0.29719 & 0.792 & Increasing & 0.792 & LVA & & \\
\hline 15 & HRV & 0.57283 & 1.146 & Decreasing & 1.146 & LVA & & \\
\hline 16 & HUN & 0.46703 & 0.934 & Increasing & 0.934 & LVA & & \\
\hline 17 & IRL & 0.21676 & 0.723 & Increasing & 0.723 & LVA & & \\
\hline 18 & ISL & 0.91145 & 1.823 & Decreasing & 1.823 & LVA & & \\
\hline 19 & ITA & 0.31198 & 0.832 & Increasing & 0.832 & LVA & & \\
\hline 20 & LTU & 0.22429 & 0.972 & Increasing & 0.972 & LVA & & \\
\hline
\end{tabular}


Table A9. Cont.

\begin{tabular}{|c|c|c|c|c|c|c|c|c|}
\hline DMU No & DMU Name & CRS Efficiency & S1 & RTS & Benchmarks & DMU & $\lambda$ & DMU \\
\hline 21 & LVA & 1.00000 & 1.000 & Constant & 1.000 & LVA & & \\
\hline 22 & MLT & 0.13725 & 0.869 & Increasing & 0.869 & LVA & & \\
\hline 23 & NLD & 0.11048 & 0.589 & Increasing & 0.589 & LVA & & \\
\hline 24 & NOR & 0.65449 & 1.745 & Decreasing & 1.745 & LVA & & \\
\hline 25 & POL & 0.32101 & 0.749 & Increasing & 0.749 & LVA & & \\
\hline 26 & PRT & 0.41260 & 0.963 & Increasing & 0.963 & LVA & & \\
\hline 27 & ROU & 0.37347 & 0.996 & Increasing & 0.996 & LVA & & \\
\hline 28 & SVK & 0.27950 & 0.838 & Increasing & 0.838 & LVA & & \\
\hline 29 & SVN & 0.42390 & 0.989 & Increasing & 0.447 & FIN & 0.542 & LVA \\
\hline 30 & SWE & 0.86584 & 1.154 & Decreasing & 0.323 & FIN & 0.832 & LVA \\
\hline
\end{tabular}

Note: DMU—decision-making unit, CRS—Constant Returns to Scale, RTS—Returns to Scale, AUT-Austria, BEL-Belgium, BGRBulgaria, CHE-Switzerland, CYP-Cyprus, CZE-The Czech Republic, DEU—Germany, DNK—Denmark, ESP-Spain, EST—Estonia, FIN-Finland, FRA-ZZZ, GBR-United Kingdom, BEL-Belgium, GRC—Greece, HRV-Croatia, HUN-Hungary, IRL-Ireland, ISLIceland, ITA—Italy, LTU—Lithuania, LVA—Latvia, MLT—Malta, NLD—Netherlands, NOR—Norway, POL—Poland, PRT—Portugal, ROU—Romania, SVK-Slovak Republic, SVN-Slovenia.

Table A10. Input-Oriented VRS Efficiency of EPS in 2010. Source: Authors' calculations in DEAFrontier.

\begin{tabular}{|c|c|c|c|c|c|c|c|c|}
\hline DMU No. & DMU Name & VRS Efficiency & Benchmarks & DMU & $\lambda$ & DMU & $\lambda$ & DMU \\
\hline 1 & AUT & 0.69363 & 0.247 & ISL & 0.333 & LVA & 0.419 & SWE \\
\hline 2 & BEL & 0.25000 & 0.891 & CYP & 0.012 & FIN & 0.097 & LVA \\
\hline 3 & BGR & 0.42857 & 0.553 & CYP & 0.447 & LVA & & \\
\hline 4 & $\mathrm{CHE}$ & 0.52081 & 0.042 & ISL & 0.958 & LVA & & \\
\hline 5 & CYP & 1.00000 & 1.000 & CYP & & & & \\
\hline 6 & CZE & 0.30000 & 0.712 & CYP & 0.288 & FIN & & \\
\hline 7 & DEU & 0.50000 & 0.662 & CYP & 0.110 & FIN & 0.228 & LVA \\
\hline 8 & DNK & 0.75000 & 0.198 & CYP & 0.494 & FIN & 0.308 & LVA \\
\hline 9 & ESP & 0.27273 & 0.413 & CYP & 0.587 & LVA & & \\
\hline 10 & EST & 1.00000 & 0.375 & CYP & 0.625 & FIN & & \\
\hline 11 & FIN & 1.00000 & 1.000 & FIN & & & & \\
\hline 12 & FRA & 0.30000 & 0.513 & CYP & 0.487 & LVA & & \\
\hline 13 & GBR & 0.30000 & 0.898 & CYP & 0.102 & LVA & & \\
\hline 14 & GRC & 0.37500 & 0.677 & CYP & 0.012 & FIN & 0.311 & LVA \\
\hline 15 & HRV & 0.58851 & 0.177 & ISL & 0.823 & LVA & & \\
\hline 16 & HUN & 0.50000 & 1.000 & LVA & & & & \\
\hline 17 & IRL & 0.30000 & 0.590 & CYP & 0.405 & FIN & 0.005 & LVA \\
\hline 18 & ISL & 1.00000 & 1.000 & ISL & & & & \\
\hline 19 & ITA & 0.37500 & 0.446 & CYP & 0.210 & FIN & 0.344 & LVA \\
\hline 20 & LTU & 0.23077 & 1.000 & LVA & & & & \\
\hline 21 & LVA & 1.00000 & 1.000 & LVA & & & & \\
\hline 22 & MLT & 0.15789 & 0.755 & CYP & 0.245 & LVA & & \\
\hline 23 & NLD & 0.18750 & 0.720 & CYP & 0.280 & FIN & & \\
\hline 24 & NOR & 1.00000 & 1.000 & NOR & & & & \\
\hline 25 & POL & 0.42857 & 0.784 & CYP & 0.216 & FIN & & \\
\hline 26 & PRT & 0.42857 & 0.038 & CYP & 0.962 & LVA & & \\
\hline 27 & ROU & 0.37500 & 1.000 & LVA & & & & \\
\hline 28 & SVK & 0.33333 & 0.484 & CYP & 0.180 & FIN & 0.336 & LVA \\
\hline 29 & SVN & 0.42857 & 0.025 & CYP & 0.462 & FIN & 0.513 & LVA \\
\hline 30 & SWE & 1.00000 & 1.000 & SWE & & & & \\
\hline
\end{tabular}

Note: DMU—decision-making unit, VRS—Variable Returns to Scale, AUT—Austria, BEL-Belgium, BGR—Bulgaria, CHE-Switzerland, CYP-Cyprus, CZE-The Czech Republic, DEU—Germany, DNK—Denmark, ESP—Spain, EST—Estonia, FIN—Finland, FRA—ZZZ, GBR-United Kingdom, BEL-Belgium, GRC - Greece, HRV—Croatia, HUN—Hungary, IRL-Ireland, ISL-Iceland, ITA-Italy, LTU— Lithuania, LVA—Latvia, MLT—Malta, NLD—Netherlands, NOR—Norway, POL—Poland, PRT—Portugal, ROU—Romania, SVK—Slovak Republic, SVN-Slovenia. 
Table A11. Input-Oriented CRS Efficiency of EPS in 2009. Source: Authors' calculations in DEAFrontier.

\begin{tabular}{|c|c|c|c|c|c|c|}
\hline DMU No & DMU Name & CRS Efficiency & S1 & RTS & Benchmarks & DMU \\
\hline 1 & AUT & 0.44564 & 1.114 & Decreasing & 1.114 & LVA \\
\hline 2 & BEL & 0.13347 & 0.667 & Increasing & 0.667 & LVA \\
\hline 3 & BGR & 0.15735 & 0.865 & Increasing & 0.865 & LVA \\
\hline 4 & $\mathrm{CHE}$ & 0.29491 & 0.885 & Increasing & 0.885 & LVA \\
\hline 5 & CYP & 0.51390 & 0.771 & Increasing & 0.771 & LVA \\
\hline 6 & CZE & 0.18267 & 0.639 & Increasing & 0.639 & LVA \\
\hline 7 & DEU & 0.23008 & 0.690 & Increasing & 0.690 & LVA \\
\hline 8 & DNK & 0.36634 & 0.733 & Increasing & 0.733 & LVA \\
\hline 9 & ESP & 0.15231 & 0.838 & Increasing & 0.838 & LVA \\
\hline 10 & EST & 0.24549 & 0.982 & Increasing & 0.982 & LVA \\
\hline 11 & FIN & 0.90606 & 1.359 & Decreasing & 1.359 & LVA \\
\hline 12 & FRA & 0.17644 & 0.882 & Increasing & 0.882 & LVA \\
\hline 13 & GBR & 0.15208 & 0.760 & Increasing & 0.760 & LVA \\
\hline 14 & GRC & 0.15998 & 0.720 & Increasing & 0.720 & LVA \\
\hline 15 & HRV & 0.26057 & 0.912 & Increasing & 0.912 & LVA \\
\hline 16 & HUN & 0.30546 & 0.916 & Increasing & 0.916 & LVA \\
\hline 17 & IRL & 0.12417 & 0.683 & Increasing & 0.683 & LVA \\
\hline 18 & ISL & 0.52197 & 1.566 & Decreasing & 1.566 & LVA \\
\hline 19 & ITA & 0.20116 & 0.805 & Increasing & 0.805 & LVA \\
\hline 20 & LTU & 0.16113 & 0.967 & Increasing & 0.967 & LVA \\
\hline 21 & LVA & 1.00000 & 1.000 & Constant & 1.000 & LVA \\
\hline 22 & MLT & 0.11327 & 0.850 & Increasing & 0.850 & LVA \\
\hline 23 & NLD & 0.06958 & 0.591 & Increasing & 0.591 & LVA \\
\hline 24 & NOR & 0.37809 & 1.512 & Decreasing & 1.512 & LVA \\
\hline 25 & POL & 0.21348 & 0.747 & Increasing & 0.747 & LVA \\
\hline 26 & PRT & 0.30000 & 0.900 & Increasing & 0.900 & LVA \\
\hline 27 & ROU & 0.32136 & 0.964 & Increasing & 0.964 & LVA \\
\hline 28 & SVK & 0.18486 & 0.832 & Increasing & 0.832 & LVA \\
\hline 29 & SVN & 0.25572 & 1.151 & Decreasing & 1.151 & LVA \\
\hline 30 & SWE & 0.50959 & 1.274 & Decreasing & 1.274 & LVA \\
\hline
\end{tabular}

Note: DMU—decision-making unit, CRS—Constant Returns to Scale, RTS—Returns to Scale, AUT-Austria, BEL-Belgium, BGRBulgaria, CHE-Switzerland, CYP-Cyprus, CZE-The Czech Republic, DEU-Germany, DNK-Denmark, ESP-Spain, EST-Estonia, FIN-Finland, FRA-ZZZ, GBR-United Kingdom, BEL-Belgium, GRC - Greece, HRV-Croatia, HUN-Hungary, IRL-Ireland, ISLIceland, ITA-Italy, LTU_Lithuania, LVA-Latvia, MLT-Malta, NLD—Netherlands, NOR-Norway, POL-Poland, PRT-Portugal, ROU—Romania, SVK-Slovak Republic, SVN-Slovenia.

Table A12. Input-Oriented VRS Efficiency of EPS in 2009. Source: Authors' calculations in DEAFrontier.

\begin{tabular}{|c|c|c|c|c|c|c|c|c|}
\hline DMU No. & DMU Name & VRS Efficiency & Benchmarks & DMU & $\lambda$ & DMU & $\lambda$ & DMU \\
\hline 1 & AUT & 0.62528 & 0.080 & ISL & 0.786 & LVA & 0.134 & NOR \\
\hline 2 & BEL & 0.20000 & 1.000 & LVA & & & & \\
\hline 3 & BGR & 0.18182 & 1.000 & LVA & & & & \\
\hline 4 & $\mathrm{CHE}$ & 0.33333 & 1.000 & LVA & & & & \\
\hline 5 & CYP & 0.66667 & 1.000 & LVA & & & & \\
\hline 6 & CZE & 0.28571 & 1.000 & LVA & & & & \\
\hline 7 & DEU & 0.33333 & 1.000 & LVA & & & & \\
\hline 8 & DNK & 0.50000 & 1.000 & LVA & & & & \\
\hline 9 & ESP & 0.18182 & 1.000 & LVA & & & & \\
\hline 10 & EST & 0.25000 & 1.000 & LVA & & & & \\
\hline 11 & FIN & 1.00000 & 1.000 & FIN & & & & \\
\hline 12 & FRA & 0.20000 & 1.000 & LVA & & & & \\
\hline 13 & GBR & 0.20000 & 1.000 & LVA & & & & \\
\hline 14 & GRC & 0.22222 & 1.000 & LVA & & & & \\
\hline 15 & HRV & 0.28571 & 1.000 & LVA & & & & \\
\hline 16 & HUN & 0.33333 & 1.000 & LVA & & & & \\
\hline 17 & IRL & 0.18182 & 1.000 & LVA & & & & \\
\hline 18 & ISL & 1.00000 & 1.000 & ISL & & & & \\
\hline
\end{tabular}


Table A12. Cont.

\begin{tabular}{|c|c|c|c|c|c|c|c|c|}
\hline DMU No. & DMU Name & VRS Efficiency & Benchmarks & DMU & $\lambda$ & DMU & $\lambda$ & DMU \\
\hline 19 & ITA & 0.25000 & 1.000 & LVA & & & & \\
\hline 20 & LTU & 0.16667 & 1.000 & LVA & & & & \\
\hline 21 & LVA & 1.00000 & 1.000 & LVA & & & & \\
\hline 22 & MLT & 0.13333 & 1.000 & LVA & & & & \\
\hline 23 & NLD & 0.11765 & 1.000 & LVA & & & & \\
\hline 24 & NOR & 1.00000 & 1.000 & NOR & & & & \\
\hline 25 & POL & 0.28571 & 1.000 & LVA & & & & \\
\hline 26 & PRT & 0.33333 & 1.000 & LVA & & & & \\
\hline 27 & ROU & 0.33333 & 1.000 & LVA & & & & \\
\hline 28 & SVK & 0.22222 & 1.000 & LVA & & & & \\
\hline 29 & SVN & 0.26886 & 0.420 & FIN & 0.580 & LVA & & \\
\hline 30 & SWE & 1.00000 & 1.000 & SWE & & & & \\
\hline
\end{tabular}

Note: DMU—decision-making unit, VRS—Variable Returns to Scale, AUT—Austria, BEL—Belgium, BGR—Bulgaria, CHE—Switzerland, CYP-Cyprus, CZE-The Czech Republic, DEU—Germany, DNK—Denmark, ESP-Spain, EST—Estonia, FIN-Finland, FRA-ZZZ, GBR-United Kingdom, BEL-Belgium, GRC - Greece, HRV—Croatia, HUN-Hungary, IRL—Ireland, ISL-Iceland, ITA-Italy, LTU— Lithuania, LVA—Latvia, MLT—Malta, NLD—Netherlands, NOR—Norway, POL—Poland, PRT—Portugal, ROU—Romania, SVK—Slovak Republic, SVN-Slovenia.

Table A13. Input-Oriented CRS Efficiency of EPS in 2008. Source: Authors' calculations in DEAFrontier.

\begin{tabular}{|c|c|c|c|c|c|c|c|c|c|c|}
\hline DMU No & DMU Name & CRS Efficiency & S1 & RTS & Benchmarks & DMU & $\lambda$ & DMU & $\lambda$ & DMU \\
\hline 1 & AUT & 0.93336 & 1.079 & Decreasing & 0.602 & FIN & 0.398 & ISL & 0.079 & NOR \\
\hline 2 & BEL & 0.27036 & 0.901 & Increasing & 0.760 & CYP & 0.141 & FIN & & \\
\hline 3 & BGR & 0.48275 & 1.126 & Decreasing & 0.887 & CYP & 0.240 & FIN & & \\
\hline 4 & $\mathrm{CHE}$ & 0.90267 & 1.444 & Decreasing & 1.384 & FIN & 0.060 & ISL & & \\
\hline 5 & CYP & 1.00000 & 1.000 & Constant & 1.000 & CYP & & & & \\
\hline 6 & CZE & 0.27916 & 0.837 & Increasing & 0.494 & CYP & 0.344 & FIN & & \\
\hline 7 & DEU & 0.58215 & 0.970 & Increasing & 0.565 & CYP & 0.405 & FIN & & \\
\hline 8 & DNK & 0.65958 & 1.099 & Decreasing & 0.334 & CYP & 0.766 & FIN & & \\
\hline 9 & ESP & 0.34784 & 1.159 & Decreasing & 0.608 & CYP & 0.552 & FIN & & \\
\hline 10 & EST & 0.21892 & 0.803 & Increasing & 0.106 & CYP & 0.697 & FIN & & \\
\hline 11 & FIN & 1.00000 & 1.000 & Constant & 1.000 & FIN & & & & \\
\hline 12 & FRA & 0.40781 & 1.223 & Decreasing & 0.869 & CYP & 0.355 & FIN & & \\
\hline 13 & GBR & 0.33132 & 0.994 & Increasing & 0.843 & CYP & 0.151 & FIN & & \\
\hline 14 & GRC & 0.33244 & 0.997 & Increasing & 0.749 & CYP & 0.249 & FIN & & \\
\hline 15 & HRV & 0.71585 & 1.432 & Decreasing & 0.159 & CYP & 1.273 & FIN & & \\
\hline 16 & HUN & 0.60053 & 1.201 & Decreasing & 1.046 & CYP & 0.156 & FIN & & \\
\hline 17 & IRL & 0.25863 & 0.948 & Increasing & 0.619 & CYP & 0.329 & FIN & & \\
\hline 18 & ISL & 1.00000 & 1.000 & Constant & 1.000 & ISL & & & & \\
\hline 19 & ITA & 0.42060 & 1.122 & Decreasing & 0.610 & CYP & 0.512 & FIN & & \\
\hline 20 & LTU & 0.47325 & 1.262 & Decreasing & 1.065 & CYP & 0.197 & FIN & & \\
\hline 21 & LVA & 0.61823 & 1.586 & Decreasing & 1.524 & FIN & 0.062 & ISL & & \\
\hline 22 & MLT & 0.22322 & 1.116 & Decreasing & 1.116 & CYP & & & & \\
\hline 23 & NLD & 0.16963 & 0.848 & Increasing & 0.606 & CYP & 0.242 & FIN & & \\
\hline 24 & NOR & 1.00000 & 1.000 & Constant & 1.000 & NOR & & & & \\
\hline 25 & POL & 0.43601 & 1.017 & Decreasing & 0.810 & CYP & 0.207 & FIN & & \\
\hline 26 & PRT & 0.58144 & 1.357 & Decreasing & 0.463 & CYP & 0.894 & FIN & & \\
\hline 27 & ROU & 0.81943 & 1.366 & Decreasing & 0.632 & CYP & 0.734 & FIN & & \\
\hline 28 & SVK & 0.43046 & 1.148 & Decreasing & 0.712 & CYP & 0.436 & FIN & & \\
\hline 29 & SVN & 0.41294 & 1.101 & Decreasing & 0.342 & CYP & 0.759 & FIN & & \\
\hline 30 & SWE & 0.89140 & 1.450 & Decreasing & 1.414 & FIN & 0.036 & ISL & & \\
\hline
\end{tabular}

Note: DMU—decision-making unit, CRS—Constant Returns to Scale, RTS—Returns to Scale, AUT_-Austria, BEL-Belgium, BGR_Bulgaria, CHE—Switzerland, CYP_Cyprus, CZE—The Czech Republic, DEU—Germany, DNK-Denmark, ESP-Spain, EST_Estonia, FIN-Finland, FRA-ZZZ, GBR_-United Kingdom, BEL_Belgium, GRC_-Greece, HRV-Croatia, HUN-Hungary, IRL-Ireland, ISLIceland, ITA-Italy, LTU—Lithuania, LVA-Latvia, MLT-Malta, NLD—Netherlands, NOR-Norway, POL-Poland, PRT-Portugal, ROU-Romania, SVK-Slovak Republic, SVN-Slovenia. 
Table A14. Input-Oriented VRS Efficiency of EPS in 2008. Source: Authors' calculations in DEAFrontier.

\begin{tabular}{|c|c|c|c|c|c|c|c|c|c|c|}
\hline DMU No. & DMU Name & VRS Efficiency & Benchmarks & DMU & $\lambda$ & DMU & $\lambda$ & DMU & $\lambda$ & DMU \\
\hline 1 & AUT & 0.98456 & 0.255 & FIN & 0.235 & ISL & 0.197 & NOR & 0.314 & SWE \\
\hline 2 & BEL & 0.30000 & 0.860 & CYP & 0.140 & FIN & & & & \\
\hline 3 & BGR & 0.54671 & 0.587 & CYP & 0.129 & ROU & 0.284 & SWE & & \\
\hline 4 & $\mathrm{CHE}$ & 1.00000 & 1.000 & $\mathrm{CHE}$ & & & & & & \\
\hline 5 & CYP & 1.00000 & 1.000 & CYP & & & & & & \\
\hline 6 & CZE & 0.33333 & 0.712 & CYP & 0.288 & FIN & & & & \\
\hline 7 & DEU & 0.60000 & 0.595 & CYP & 0.405 & FIN & & & & \\
\hline 8 & DNK & 0.68242 & 0.206 & $\mathrm{CHE}$ & 0.348 & CYP & 0.446 & FIN & & \\
\hline 9 & ESP & 0.36732 & 0.050 & $\mathrm{CHE}$ & 0.623 & CYP & 0.040 & FIN & 0.287 & SWE \\
\hline 10 & EST & 0.27273 & 0.371 & CYP & 0.629 & FIN & & & & \\
\hline 11 & FIN & 1.00000 & 1.000 & FIN & & & & & & \\
\hline 12 & FRA & 0.49154 & 0.288 & CYP & 0.609 & ROU & 0.103 & SWE & & \\
\hline 13 & GBR & 0.33333 & 0.849 & CYP & 0.151 & FIN & & & & \\
\hline 14 & GRC & 0.33333 & 0.751 & CYP & 0.249 & FIN & & & & \\
\hline 15 & HRV & 0.82054 & 0.582 & $\mathrm{CHE}$ & 0.038 & CYP & 0.264 & ROU & 0.117 & SWE \\
\hline 16 & HUN & 0.75449 & 0.237 & CYP & 0.763 & ROU & & & & \\
\hline 17 & IRL & 0.27273 & 0.403 & CYP & 0.597 & FIN & & & & \\
\hline 18 & ISL & 1.00000 & 1.000 & ISL & & & & & & \\
\hline 19 & ITA & 0.43833 & 0.202 & $\mathrm{CHE}$ & 0.626 & CYP & 0.121 & FIN & 0.052 & SWE \\
\hline 20 & LTU & 0.64974 & 0.066 & LVA & 0.823 & ROU & 0.112 & SWE & & \\
\hline 21 & LVA & 1.00000 & 1.000 & LVA & & & & & & \\
\hline 22 & MLT & 0.26909 & 0.482 & CYP & 0.518 & ROU & & & & \\
\hline 23 & NLD & 0.20000 & 1.000 & FIN & & & & & & \\
\hline 24 & NOR & 1.00000 & 1.000 & NOR & & & & & & \\
\hline 25 & POL & 0.44337 & 0.782 & CYP & 0.166 & FIN & 0.052 & SWE & & \\
\hline 26 & PRT & 0.68058 & 0.104 & $\mathrm{CHE}$ & 0.118 & CYP & 0.571 & ROU & 0.206 & SWE \\
\hline 27 & ROU & 1.00000 & 1.000 & ROU & & & & & & \\
\hline 28 & SVK & 0.47314 & 0.571 & CYP & 0.036 & FIN & 0.393 & SWE & & \\
\hline 29 & SVN & 0.45050 & 0.181 & CYP & 0.517 & FIN & 0.302 & SWE & & \\
\hline 30 & SWE & 1.00000 & 1.000 & SWE & & & & & & \\
\hline
\end{tabular}

Note: DMU—decision-making unit, VRS—Variable Returns to Scale, AUT—Austria, BEL—Belgium, BGR—Bulgaria, CHE-Switzerland, CYP-Cyprus, CZE-The Czech Republic, DEU-Germany, DNK-Denmark, ESP-Spain, EST-Estonia, FIN-Finland, FRA-ZZZ, GBR-United Kingdom, BEL—Belgium, GRC - Greece, HRV-Croatia, HUN—Hungary, IRL—Ireland, ISL-Iceland, ITA-Italy, LTULithuania, LVA—Latvia, MLT—Malta, NLD—Netherlands, NOR—Norway, POL—Poland, PRT—Portugal, ROU—Romania, SVK—Slovak Republic, SVN-Slovenia.

Table A15. Input-Oriented CRS Efficiency of EPS in 2007. Source: Authors' calculations in DEAFrontier.

\begin{tabular}{ccccccccccc}
\hline DMU No & DMU Name & CRS Efficiency & S1 & RTS & Benchmarks & DMU & $\lambda$ & DMU & $\lambda$ & DMU \\
\hline 1 & AUT & 0.97636 & 1.129 & Decreasing & 0.631 & FIN & 0.483 & ISL & 0.015 & NOR \\
2 & BEL & 0.33746 & 0.889 & Increasing & 0.754 & CYP & 0.104 & FIN & 0.032 & ROU \\
3 & BGR & 0.27985 & 1.119 & Decreasing & 0.880 & CYP & 0.240 & FIN & & \\
4 & CHE & 0.82510 & 1.118 & Decreasing & 0.201 & FIN & 0.339 & ISL & 0.579 & ROU \\
5 & CYP & 1.00000 & 1.000 & Constant & 1.000 & CYP & & & & \\
6 & CZE & 0.24602 & 0.820 & Increasing & 0.470 & CYP & 0.350 & FIN & \\
7 & DEU & 0.59422 & 0.907 & Increasing & 0.408 & CYP & 0.247 & FIN & 0.251 & ROU \\
8 & DNK & 0.65133 & 0.773 & Increasing & 0.083 & ISL & 0.689 & ROU & & \\
9 & ESP & 0.33710 & 0.968 & Increasing & 0.261 & CYP & 0.239 & FIN & 0.468 & ROU \\
10 & EST & 0.26885 & 0.807 & Increasing & 0.108 & CYP & 0.699 & FIN & \\
11 & FIN & 1.00000 & 1.000 & Constant & 1.000 & FIN & & & \\
12 & FRA & 0.40205 & 1.098 & Decreasing & 0.665 & CYP & 0.107 & FIN & 0.325 & ROU \\
13 & GBR & 0.32291 & 0.905 & Increasing & 0.714 & CYP & 0.191 & ROU & \\
14 & GRC & 0.37007 & 0.971 & Increasing & 0.722 & CYP & 0.202 & FIN & 0.047 & ROU \\
15 & HRV & 0.71689 & 1.055 & Decreasing & 0.182 & FIN & 0.131 & ISL & 0.742 & ROU \\
16 & HUN & 0.58349 & 1.107 & Decreasing & 0.928 & CYP & 0.001 & FIN & 0.179 & ROU \\
17 & IRL & 0.26997 & 0.771 & Increasing & 0.386 & CYP & 0.386 & ROU &
\end{tabular}


Table A15. Cont.

\begin{tabular}{ccccccccccc}
\hline DMU No & DMU Name & CRS Efficiency & S1 & RTS & Benchmarks & DMU & $\lambda$ & DMU & $\boldsymbol{\lambda}$ & DMU \\
\hline 19 & ITA & 0.46820 & 0.954 & Increasing & 0.382 & CYP & 0.155 & FIN & 0.416 & ROU \\
20 & LTU & 0.41789 & 1.254 & Decreasing & 1.056 & CYP & 0.197 & FIN & & \\
21 & LVA & 0.59505 & 1.323 & Decreasing & 0.552 & FIN & 0.307 & ISL & 0.464 & ROU \\
22 & MLT & 0.19987 & 1.066 & Decreasing & 1.066 & CYP & & & & \\
23 & NLD & 0.16726 & 0.744 & Increasing & 0.467 & CYP & 0.277 & ROU & \\
24 & NOR & 1.00000 & 1.000 & Constant & 1.000 & NOR & & & & \\
25 & POL & 0.51142 & 1.023 & Decreasing & 0.819 & CYP & 0.204 & FIN & \\
26 & PRT & 0.69437 & 1.047 & Decreasing & 0.199 & FIN & 0.090 & ISL & 0.758 & ROU \\
27 & ROU & 1.00000 & 1.000 & Constant & 1.000 & ROU & & & \\
28 & SVK & 0.51139 & 1.086 & Decreasing & 0.454 & CYP & 0.312 & FIN & 0.321 & ROU \\
29 & SVN & 0.44574 & 1.181 & Decreasing & 0.428 & CYP & 0.728 & FIN & 0.024 & ROU \\
30 & SWE & 0.97809 & 1.331 & Decreasing & 0.817 & FIN & 0.192 & ISL & 0.322 & ROU \\
\hline
\end{tabular}

Note: DMU—decision-making unit, CRS—Constant Returns to Scale, RTS—Returns to Scale, AUT-Austria, BEL-Belgium, BGR_Bulgaria, CHE—Switzerland, CYP-Cyprus, CZE-The Czech Republic, DEU—Germany, DNK-Denmark, ESP-Spain, EST-Estonia, FIN-Finland, FRA-ZZZ, GBR-United Kingdom, BEL-Belgium, GRC—Greece, HRV-Croatia, HUN—Hungary, IRL-Ireland, ISLIceland, ITA-Italy, LTU_Lithuania, LVA-Latvia, MLT-Malta, NLD-Netherlands, NOR-Norway, POL-Poland, PRT-Portugal, ROU-Romania, SVK-Slovak Republic, SVN-Slovenia.

Table A16. Input-Oriented VRS Efficiency of EPS in 2007. Source: Authors' calculations in DEAFrontier.

\begin{tabular}{|c|c|c|c|c|c|c|c|c|c|c|}
\hline DMU No. & DMU Name & VRS Efficiency & Benchmarks & DMU & $\lambda$ & DMU & $\lambda$ & DMU & $\lambda$ & DMU \\
\hline 1 & AUT & 0.99916 & 0.231 & FIN & 0.203 & ISL & 0.255 & NOR & 0.310 & SWE \\
\hline 2 & BEL & 0.37500 & 0.528 & CYP & 0.472 & FIN & & & & \\
\hline 3 & BGR & 0.29688 & 0.253 & CYP & 0.184 & FIN & 0.563 & ROU & & \\
\hline 4 & $\mathrm{CHE}$ & 0.96330 & 0.032 & ISL & 0.187 & LVA & 0.781 & SWE & & \\
\hline 5 & CYP & 1.00000 & 1.000 & CYP & & & & & & \\
\hline 6 & CZE & 0.30000 & 0.019 & CYP & 0.981 & FIN & & & & \\
\hline 7 & DEU & 0.60692 & 0.531 & CYP & 0.435 & FIN & 0.035 & ROU & & \\
\hline 8 & DNK & 0.66983 & 0.078 & CYP & 0.573 & FIN & 0.349 & ROU & & \\
\hline 9 & ESP & 0.33930 & 0.303 & CYP & 0.304 & FIN & 0.393 & ROU & & \\
\hline 10 & EST & 0.33333 & 1.000 & FIN & & & & & & \\
\hline 11 & FIN & 1.00000 & 1.000 & FIN & & & & & & \\
\hline 12 & FRA & 0.42064 & 0.152 & CYP & 0.062 & FIN & 0.786 & ROU & & \\
\hline 13 & GBR & 0.33333 & 0.790 & CYP & 0.210 & FIN & & & & \\
\hline 14 & GRC & 0.37500 & 0.727 & CYP & 0.273 & FIN & & & & \\
\hline 15 & HRV & 0.71995 & 0.045 & FIN & 0.099 & ISL & 0.688 & ROU & 0.168 & SWE \\
\hline 16 & HUN & 0.62681 & 0.239 & CYP & 0.761 & ROU & & & & \\
\hline 17 & IRL & 0.30000 & 0.666 & CYP & 0.334 & FIN & & & & \\
\hline 18 & ISL & 1,00000 & 1.000 & ISL & & & & & & \\
\hline 19 & ITA & 0.47269 & 0.443 & CYP & 0.248 & FIN & 0.309 & ROU & & \\
\hline 20 & LTU & 0.55402 & 0.191 & LVA & 0.777 & ROU & 0.032 & SWE & & \\
\hline 21 & LVA & 1.00000 & 1.000 & LVA & & & & & & \\
\hline 22 & MLT & 0,20988 & 0.642 & CYP & 0.358 & ROU & & & & \\
\hline 23 & NLD & 0.20000 & 0.761 & CYP & 0.239 & FIN & & & & \\
\hline 24 & NOR & 1.00000 & 1.000 & NOR & & & & & & \\
\hline 25 & POL & 0.51793 & 0.699 & CYP & 0.194 & FIN & 0.108 & $\mathrm{ROU}$ & & \\
\hline 26 & PRT & 0.69694 & 0.084 & FIN & 0.062 & ISL & 0.713 & ROU & 0.141 & SWE \\
\hline 27 & ROU & 1.00000 & 1.000 & $\mathrm{ROU}$ & & & & & & \\
\hline 28 & SVK & 0.53250 & 0.000 & CYP & 0.272 & FIN & 0.727 & ROU & & \\
\hline 29 & SVN & 0.49278 & 0.422 & FIN & 0.213 & ROU & 0.365 & SWE & & \\
\hline 30 & SWE & 1.00000 & 1.000 & SWE & & & & & & \\
\hline
\end{tabular}

Note: DMU—decision-making unit, VRS—Variable Returns to Scale, AUT_Austria, BEL_Belgium, BGR—Bulgaria, CHE-Switzerland, CYP-Cyprus, CZE-The Czech Republic, DEU—Germany, DNK-Denmark, ESP-Spain, EST-Estonia, FIN-Finland, FRA-ZZZ, GBR-United Kingdom, BEL-Belgium, GRC-Greece, HRV-Croatia, HUN-Hungary, IRL-Ireland, ISL-Iceland, ITA-Italy, LTULithuania, LVA—Latvia, MLT-Malta, NLD—Netherlands, NOR_Norway, POL_Poland, PRT-Portugal, ROU—Romania, SVK-Slovak Republic, SVN-Slovenia. 
Table A17. Input-Oriented CRS Efficiency of EPS in 2006. Source: Authors' calculations in DEAFrontier.

\begin{tabular}{|c|c|c|c|c|c|c|c|c|c|c|}
\hline DMU No & DMU Name & CRS Efficiency & S1 & RTS & Benchmarks & DMU & $\lambda$ & DMU & $\lambda$ & DMU \\
\hline 1 & AUT & 0.87772 & 1.052 & Decreasing & 0.326 & FIN & 0.505 & NOR & 0.222 & ROU \\
\hline 2 & BEL & 0.32736 & 0.868 & Increasing & 0.742 & CYP & 0.113 & FIN & 0.014 & $\mathrm{ROU}$ \\
\hline 3 & BGR & 0.28699 & 1.110 & Decreasing & 0.776 & CYP & 0.221 & FIN & 0.113 & $\mathrm{ROU}$ \\
\hline 4 & $\mathrm{CHE}$ & 0.63569 & 1.038 & Decreasing & 0.008 & FIN & 0.305 & NOR & 0.726 & $\mathrm{ROU}$ \\
\hline 5 & CYP & 1.00000 & 1.000 & Constant & 1.000 & CYP & & & & \\
\hline 6 & CZE & 0.22767 & 0.835 & Increasing & 0.490 & CYP & 0.345 & FIN & & \\
\hline 7 & DEU & 0.47831 & 0.910 & Increasing & 0.506 & CYP & 0.265 & FIN & 0.139 & ROU \\
\hline 8 & DNK & 0.48072 & 0.732 & Increasing & 0.043 & CYP & 0.689 & ROU & & \\
\hline 9 & ESP & 0.33430 & 0.989 & Increasing & 0.370 & CYP & 0.241 & FIN & 0.377 & $\mathrm{ROU}$ \\
\hline 10 & EST & 0.34704 & 0.925 & Increasing & 0.266 & CYP & 0.660 & FIN & & \\
\hline 11 & FIN & 1.00000 & 1.000 & Constant & 1.000 & FIN & & & & \\
\hline 12 & FRA & 0.39199 & 1.088 & Decreasing & 0.707 & CYP & 0.118 & FIN & 0.263 & ROU \\
\hline 13 & GBR & 0.31346 & 0.888 & Increasing & 0.732 & CYP & 0.157 & ROU & & \\
\hline 14 & GRC & 0.38195 & 0.928 & Increasing & 0.481 & CYP & 0.175 & FIN & 0.272 & ROU \\
\hline 15 & HRV & 0.75373 & 1.073 & Decreasing & 0.058 & FIN & 0.287 & NOR & 0.728 & ROU \\
\hline 16 & HUN & 0.48546 & 1.090 & Decreasing & 0.947 & CYP & 0.013 & FIN & 0.129 & ROU \\
\hline 17 & IRL & 0.29855 & 0.792 & Increasing & 0.481 & CYP & 0.311 & ROU & & \\
\hline 18 & ISL & 0.89068 & 1.093 & Decreasing & 0.970 & NOR & 0.123 & ROU & & \\
\hline 19 & ITA & 0.46457 & 0.945 & Increasing & 0.373 & CYP & 0.156 & FIN & 0.416 & ROU \\
\hline 20 & LTU & 0.46807 & 1.248 & Decreasing & 1.052 & CYP & 0.197 & FIN & & \\
\hline 21 & LVA & 0.72053 & 1.278 & Decreasing & 0.357 & FIN & 0.289 & NOR & 0.632 & ROU \\
\hline 22 & MLT & 0.21329 & 1.066 & Decreasing & 1.065 & CYP & 0.001 & ROU & & \\
\hline 23 & NLD & 0.16672 & 0.741 & Increasing & 0.463 & CYP & 0.278 & ROU & & \\
\hline 24 & NOR & 1.00000 & 1.000 & Constant & 1.000 & NOR & & & & \\
\hline 25 & POL & 0.43208 & 1.008 & Decreasing & 0.800 & CYP & 0.208 & FIN & & \\
\hline 26 & PRT & 0.57072 & 1.029 & Decreasing & 0.156 & FIN & 0.036 & NOR & 0.836 & ROU \\
\hline 27 & ROU & 1.00000 & 1.000 & Constant & 1.000 & ROU & & & & \\
\hline 28 & SVK & 0.34496 & 1.079 & Decreasing & 0.532 & CYP & 0.333 & FIN & 0.214 & ROU \\
\hline 29 & SVN & 0.45326 & 1.152 & Decreasing & 0.267 & CYP & 0.714 & FIN & 0.171 & ROU \\
\hline 30 & SWE & 0.93231 & 1.294 & Decreasing & 0.703 & FIN & 0.187 & NOR & 0.404 & ROU \\
\hline
\end{tabular}

Note: DMU—decision-making unit, CRS—Constant Returns to Scale, RTS—Returns to Scale, AUT-Austria, BEL-Belgium, BGRBulgaria, CHE-Switzerland, CYP-Cyprus, CZE-The Czech Republic, DEU—Germany, DNK-Denmark, ESP-Spain, EST-Estonia, FIN—Finland, FRA-ZZZ, GBR—United Kingdom, BEL-Belgium, GRC—Greece, HRV—Croatia, HUN—Hungary, IRL—Ireland, ISLIceland, ITA—Italy, LTU—Lithuania, LVA—Latvia, MLT-Malta, NLD—Netherlands, NOR—Norway, POL—Poland, PRT—Portugal, ROU—Romania, SVK—Slovak Republic, SVN—Slovenia.

Table A18. Input-Oriented VRS Efficiency of EPS in 2006. Source: Authors' calculations in DEAFrontier.

\begin{tabular}{ccccccccccc}
\hline DMU No. & DMU Name & VRS Efficiency & Benchmarks & DMU & $\lambda$ & DMU & $\lambda$ & DMU & $\lambda$ & DMU \\
\hline 1 & AUT & 0.88979 & 0.201 & FIN & 0.471 & NOR & 0.150 & ROU & 0.178 & SWE \\
2 & BEL & 0.37500 & 0.462 & CYP & 0.538 & FIN & & & \\
3 & BGR & 0.30848 & 0.139 & CYP & 0.159 & FIN & 0.702 & ROU & \\
4 & CHE & 0.68601 & 0.242 & ISL & 0.034 & LVA & 0.508 & ROU & 0.216 & SWE \\
5 & CYP & 1.00000 & 1.000 & CYP & & & & & \\
6 & CZE & 0.27273 & 0.112 & CYP & 0.888 & FIN & & \\
7 & DEU & 0.50000 & 0.511 & CYP & 0.489 & FIN & & \\
8 & DNK & 0.52157 & 0.432 & CYP & 0.503 & FIN & 0.065 & NOR & \\
9 & ESP & 0.33529 & 0.421 & CYP & 0.243 & FIN & 0.017 & NOR & 0.320 & ROU \\
10 & EST & 0.37500 & 0.095 & CYP & 0.905 & FIN & & & \\
11 & FIN & 1.00000 & 1.000 & FIN & & & & \\
12 & FRA & 0.41492 & 0.197 & CYP & 0.069 & FIN & 0.734 & ROU & \\
13 & GBR & 0.33333 & 0.713 & CYP & 0.287 & FIN & & NOR \\
14 & GRC & 0.38997 & 0.691 & CYP & 0.249 & FIN & 0.060 & NOU & 0.161 & SWE \\
15 & HRV & 0.85356 & 0.192 & ISL & 0.192 & LVA & 0.455 & ROU \\
17 & HUN & 0.52583 & 0.319 & CYP & 0.681 & ROU & & &
\end{tabular}


Table A18. Cont.

\begin{tabular}{|c|c|c|c|c|c|c|c|c|c|c|}
\hline DMU No. & DMU Name & VRS Efficiency & Benchmarks & DMU & $\lambda$ & DMU & $\lambda$ & DMU & $\lambda$ & DMU \\
\hline 19 & ITA & 0.47131 & 0.617 & CYP & 0.163 & FIN & 0.079 & NOR & 0.140 & ROU \\
\hline 20 & LTU & 0.66208 & 0.432 & LVA & 0.568 & ROU & & & & \\
\hline 21 & LVA & 1.00000 & 1.000 & LVA & & & & & & \\
\hline 22 & MLT & 0.22846 & 0.573 & CYP & 0.427 & ROU & & & & \\
\hline 23 & NLD & 0.20000 & 0.702 & CYP & 0.298 & FIN & & & & \\
\hline 24 & NOR & 1.00000 & 1.000 & NOR & & & & & & \\
\hline 25 & POL & 0.43481 & 0.753 & CYP & 0.203 & FIN & 0.044 & ROU & & \\
\hline 26 & PRT & 0.57543 & 0.088 & FIN & 0.018 & NOR & 0.797 & ROU & 0.097 & SWE \\
\hline 27 & ROU & 1.00000 & 1.000 & $\mathrm{ROU}$ & & & & & & \\
\hline 28 & SVK & 0.36334 & 0.077 & CYP & 0.289 & FIN & 0.633 & ROU & & \\
\hline 29 & SVN & 0.50067 & 0.392 & FIN & 0.210 & ROU & 0.398 & SWE & & \\
\hline 30 & SWE & 1.00000 & 1.000 & SWE & & & & & & \\
\hline
\end{tabular}

Note: DMU—decision-making unit, VRS—Variable Returns to Scale, AUT-Austria, BEL-Belgium, BGR-Bulgaria, CHE-Switzerland, CYP-Cyprus, CZE-The Czech Republic, DEU-Germany, DNK-Denmark, ESP-Spain, EST-Estonia, FIN-Finland, FRA-ZZZ, GBR-United Kingdom, BEL-Belgium, GRC - Greece, HRV-Croatia, HUN-Hungary, IRL-Ireland, ISL-Iceland, ITA-Italy, LTULithuania, LVA —Latvia, MLT-Malta, NLD—Netherlands, NOR—Norway, POL_Poland, PRT—Portugal, ROU—Romania, SVK—Slovak Republic, SVN-Slovenia.

Table A19. Input-Oriented CRS Efficiency of EPS in 2005. Source: Authors' calculations in DEAFrontier.

\begin{tabular}{|c|c|c|c|c|c|c|c|c|c|c|}
\hline DMU No & DMU Name & CRS Efficiency & S1 & RTS & Benchmarks & DMU & $\lambda$ & DMU & $\lambda$ & DMU \\
\hline 1 & AUT & 0.87226 & 0.994 & Increasing & 0.369 & FIN & 0.460 & NOR & 0.165 & ROU \\
\hline 2 & BEL & 0.27260 & 0.727 & Increasing & 0.049 & FIN & 0.678 & ROU & & \\
\hline 3 & BGR & 0.41130 & 0.960 & Increasing & 0.154 & FIN & 0.805 & ROU & & \\
\hline 4 & $\mathrm{CHE}$ & 0.57691 & 1.026 & Decreasing & 0.013 & FIN & 0.320 & NOR & 0.692 & ROU \\
\hline 5 & CYP & 0.86323 & 0.863 & Increasing & 0.863 & ROU & & & & \\
\hline 6 & CZE & 0.20007 & 0.734 & Increasing & 0.309 & FIN & 0.425 & ROU & & \\
\hline 7 & DEU & 0.40590 & 0.812 & Increasing & 0.225 & FIN & 0.587 & ROU & & \\
\hline 8 & DNK & 0.39772 & 0.795 & Increasing & 0.000 & NOR & 0.795 & ROU & & \\
\hline 9 & ESP & 0.29617 & 0.888 & Increasing & 0.221 & FIN & 0.667 & ROU & & \\
\hline 10 & EST & 0.27067 & 0.812 & Increasing & 0.680 & FIN & 0.132 & ROU & & \\
\hline 11 & FIN & 1.00000 & 1.000 & Constant & 1.000 & FIN & & & & \\
\hline 12 & FRA & 0.31358 & 0.941 & Increasing & 0.063 & FIN & 0.878 & ROU & & \\
\hline 13 & GBR & 0.16722 & 0.780 & Increasing & 0.780 & ROU & & & & \\
\hline 14 & GRC & 0.41063 & 0.821 & Increasing & 0.140 & FIN & 0.681 & ROU & & \\
\hline 15 & HRV & 0.66575 & 1.051 & Decreasing & 0.074 & FIN & 0.281 & NOR & 0.696 & ROU \\
\hline 16 & HUN & 0.46977 & 0.940 & Increasing & 0.940 & ROU & & & & \\
\hline 17 & IRL & 0.23606 & 0.708 & Increasing & 0.708 & ROU & & & & \\
\hline 18 & ISL & 0.87562 & 1.075 & Decreasing & 0.968 & NOR & 0.106 & ROU & & \\
\hline 19 & ITA & 0.32211 & 0.859 & Increasing & 0.132 & FIN & 0.727 & ROU & & \\
\hline 20 & LTU & 0.52373 & 1.047 & Decreasing & 0.105 & FIN & 0.942 & ROU & & \\
\hline 21 & LVA & 0.71594 & 1.256 & Decreasing & 0.354 & FIN & 0.415 & NOR & 0.487 & ROU \\
\hline 22 & MLT & 0.19150 & 0.894 & Increasing & 0.894 & ROU & & & & \\
\hline 23 & NLD & 0.13475 & 0.674 & Increasing & 0.674 & ROU & & & & \\
\hline 24 & NOR & 1.00000 & 1.000 & Constant & 1.000 & NOR & & & & \\
\hline 25 & POL & 0.43517 & 0.870 & Increasing & 0.130 & FIN & 0.740 & ROU & & \\
\hline 26 & PRT & 0.48739 & 0.975 & Increasing & 0.199 & FIN & 0.776 & ROU & & \\
\hline 27 & ROU & 1.00000 & 1.000 & Constant & 1.000 & ROU & & & & \\
\hline 28 & SVK & 0.25941 & 0.951 & Increasing & 0.306 & FIN & 0.645 & ROU & & \\
\hline 29 & SVN & 0.39164 & 1.044 & Decreasing & 0.728 & FIN & 0.316 & ROU & & \\
\hline 30 & SWE & 0.82238 & 1.203 & Decreasing & 0.767 & FIN & 0.167 & NOR & 0.269 & ROU \\
\hline
\end{tabular}

Note: DMU—decision-making unit, CRS—Constant Returns to Scale, RTS—Returns to Scale, AUT_Austria, BEL-Belgium, BGR_Bulgaria, CHE—Switzerland, CYP—Cyprus, CZE—The Czech Republic, DEU—Germany, DNK—Denmark, ESP-Spain, EST—Estonia, FIN-Finland, FRA-ZZZ, GBR_United Kingdom, BEL-Belgium, GRC—Greece, HRV-Croatia, HUN—Hungary, IRL-Ireland, ISLIceland, ITA-Italy, LTU-Lithuania, LVA-Latvia, MLT-Malta, NLD-Netherlands, NOR-Norway, POL-Poland, PRT-Portugal, ROU-Romania, SVK-Slovak Republic, SVN-Slovenia. 
Table A20. Input-Oriented VRS Efficiency of EPS in 2005. Source: Authors' calculations in DEAFrontier.

\begin{tabular}{|c|c|c|c|c|c|c|c|c|c|c|}
\hline DMU No. & DMU Name & VRS Efficiency & Benchmarks & DMU & $\lambda$ & DMU & $\lambda$ & DMU & $\lambda$ & DMU \\
\hline 1 & AUT & 0.87399 & 0.366 & FIN & 0.457 & NOR & 0.178 & ROU & & \\
\hline 2 & BEL & 0.37500 & 0.927 & CYP & 0.068 & FIN & 0.005 & ROU & & \\
\hline 3 & BGR & 0.42857 & 0.130 & FIN & 0.870 & $\mathrm{ROU}$ & & & & \\
\hline 4 & $\mathrm{CHE}$ & 0.61707 & 0.042 & ISL & 0.117 & LVA & 0.228 & NOR & 0.613 & $\mathrm{ROU}$ \\
\hline 5 & CYP & 1.00000 & 1.000 & CYP & & & & & & \\
\hline 6 & CZE & 0.27273 & 0.712 & CYP & 0.288 & FIN & & & & \\
\hline 7 & DEU & 0.50000 & 0.696 & CYP & 0.249 & FIN & 0.055 & ROU & & \\
\hline 8 & DNK & 0.50000 & 1.000 & ROU & & & & & & \\
\hline 9 & ESP & 0.33333 & 0.152 & FIN & 0.848 & ROU & & & & \\
\hline 10 & EST & 0.33333 & 0.363 & CYP & 0.637 & FIN & & & & \\
\hline 11 & FIN & 1.00000 & 1.000 & FIN & & & & & & \\
\hline 12 & FRA & 0.33333 & 0.026 & FIN & 0.974 & ROU & & & & \\
\hline 13 & GBR & 0.21429 & 0.872 & CYP & 0.128 & FIN & & & & \\
\hline 14 & GRC & 0.50000 & 0.680 & CYP & 0.167 & FIN & 0.153 & ROU & & \\
\hline 15 & HRV & 0.73035 & 0.191 & LVA & 0.200 & NOR & 0.600 & ROU & 0.008 & SWE \\
\hline 16 & HUN & 0.50000 & 0.442 & CYP & 0.558 & ROU & & & & \\
\hline 17 & IRL & 0.33333 & 0.786 & CYP & 0.214 & ROU & & & & \\
\hline 18 & ISL & 1.00000 & 1.000 & ISL & & & & & & \\
\hline 19 & ITA & 0.37500 & 0.044 & FIN & 0.956 & ROU & & & & \\
\hline 20 & LTU & 0.69364 & 0.290 & LVA & 0.710 & ROU & & & & \\
\hline 21 & LVA & 1.00000 & 1.000 & LVA & & & & & & \\
\hline 22 & MLT & 0.21429 & 1.000 & ROU & & & & & & \\
\hline 23 & NLD & 0.20000 & 0.782 & CYP & 0.218 & ROU & & & & \\
\hline 24 & NOR & 1.00000 & 1.000 & NOR & & & & & & \\
\hline 25 & POL & 0.50000 & 0.050 & FIN & 0.950 & ROU & & & & \\
\hline 26 & PRT & 0.50000 & 0.184 & FIN & 0.816 & ROU & & & & \\
\hline 27 & ROU & 1.00000 & 1.000 & ROU & & & & & & \\
\hline 28 & SVK & 0.27273 & 0.276 & FIN & 0.724 & ROU & & & & \\
\hline 29 & SVN & 0.43808 & 0.525 & FIN & 0.222 & ROU & 0.252 & SWE & & \\
\hline 30 & SWE & 1.00000 & 1.000 & SWE & & & & & & \\
\hline
\end{tabular}

Note: DMU—decision-making unit, VRS—Variable Returns to Scale, AUT—Austria, BEL—Belgium, BGR—Bulgaria, CHE—Switzerland, CYP-Cyprus, CZE-The Czech Republic, DEU-Germany, DNK-Denmark, ESP-Spain, EST-Estonia, FIN-Finland, FRA-ZZZ, GBR-United Kingdom, BEL—Belgium, GRC - Greece, HRV—Croatia, HUN—Hungary, IRL-Ireland, ISL-Iceland, ITA-Italy, LTU— Lithuania, LVA—Latvia, MLT—Malta, NLD—Netherlands, NOR—Norway, POL—Poland, PRT—Portugal, ROU—Romania, SVK—Slovak Republic, SVN-Slovenia.

\section{References}

1. Pearce, D.; Palmer, C. Public and private spending for environmental protection: A cross-country policy analysis. Fisc. Stud. 2001, 22, 403-456. [CrossRef]

2. Ercolano, S.; Romano, O. Spending for the environment: General government expenditure trends in Europe. Soc. Indic. Res. 2018, 138, 1145-1169. [CrossRef]

3. Bernauer, T.; Koubi, V. States as providers of public goods: How does government size affect environmental quality? SSRN 2006, 14, 1-33. Available online: http:/ / ssrn.com/abstract=900487 (accessed on 12 May 2021). [CrossRef]

4. Frederik, C.; Lundström, S. Political and Economic Freedom and the Environment: The Case of $\mathrm{CO}_{2}$ Emissions; Working Paper in Economics; University of Gothenburg: Gothenburg, Sweden, 2001.

5. Lopez, R.; Galinato, G.I.; Islam, F. Fiscal spending and the environment: Theory and empirics. J. Environ. Econ. Manag. 2011, 62, 180-198. [CrossRef]

6. Halkos, G.E.; Paizanos, E.A. The effect of government expenditure on the environment: An empirical investigation. Ecol. Econ. 2013, 91, 48-56. [CrossRef]

7. Halkos, G.E.; Paizanos, E.A. The effects of fiscal policy on $\mathrm{CO}_{2}$ emissions: Evidence from the USA. Energy Policy 2016, 88, 317-328. [CrossRef]

8. Galinato, G.I.; Islam, F. The Challenge of Addressing Consumption Pollutants with Fiscal Policy; Working Paper Series WP 2014-1; Washington State University: Washington, DC, USA, 2014.

9. Xing, M.; Tan, T. Environmental attitudes and impactsof privatization on R\&D, environment and welfare in a mixed duopoly. Econ. Res.-Ekon. Istr. 2021, 34, 807-827. [CrossRef]

10. Hettige, H.; Huq, M.; Pargal, S.; Wheeler, D. Determinants of pollution abatement in developing countries. World Dev. 1996, 24, 1891-1904. [CrossRef] 
11. Ohori, S. Optimal environmental tax and level of privatization in an international duopoly. J. Regul. Econ. 2006, $29,225-233$. [CrossRef]

12. Pal, R.; Saha, B. Pollution tax, partial privatization and environment. Resour. Energy Econ. 2015, 40, 19-35. [CrossRef]

13. Chang, L.; Li, W.; Lu, X. Government engagement, environmental policy, and environmental performance: Evidence from the most polluting Chinese listed firms. Bus. Strategy Environ. 2015, 24, 1-19. [CrossRef]

14. Xing, M.; Tan, T.; Wang, X. Environmental R\&D subsidy, spillovers and privatization in a mixed duopoly. Econ. Res.-Ekon. Istr. 2019, 32, 2989-3015. [CrossRef]

15. Jordan, A.; Adelle, C. Environmental Policy in the EU: Actors, Institutions and Processes; Routledge: London, UK, 2021.

16. Destek, M.A.; Ulucak, R.; Dogan, E. Analyzing the environmental Kuznets curve for the EU countries: The role of ecological footprint. Environ. Sci. Pollut. Res. Int. 2018, 25, 29387-29396. [CrossRef] [PubMed]

17. Wach, K.; Głodowska, A.; Maciejewski, M.; Sieja, M. Europeanization processes of the EU energy policy in Visegrad countries in the years 2005-2018. Energies 2021, 14, 1802. [CrossRef]

18. Allen, M.L.; Allen, M.M.C.; Cumming, D.; Johan, S. Comparative capitalisms and energy transitions: Renewable energy in the European Union. Br. J. Manag. 2020, 32, 611-629. [CrossRef]

19. Agranoff, R.; Ballart, X. The Effects on Domestic Versus Europeanization Influences on Intergovernmental Relations: The Case of Spain; Instituto Universitario Ortega y Gasset: Madrid, Spain, 2009; pp. 1-46.

20. Solorio, I.; Jörgens, H. Contested energy transition? Europeanization and authority turns in EU renewable energy policy. J. Eur. Integr. 2020, 42, 77-93. [CrossRef]

21. Vasconcelos, J. Towards the internal energy market, how to bridge a regulatory gap and build a regulatory framework. Eur. Rev. Energy Mark. 2005, 1, 81-103.

22. Bouzarovski, S.; Petrova, S.; Sarlamanov, R. Energy poverty policies in the EU: A critical perspective. Energy Policy 2012, 49, 76-82. [CrossRef]

23. Laing, T.; Sato, M.; Grubb, M.; Comberti, C. Assessing the Effectiveness of the EU Emissions Trading System; Working Paper; Center for Climate Change Economics and Policy: London, UK, 2013; Volume 126, Available online: https://www.lse.ac.uk/ granthaminstitute/wpcontent/uploads/2014/02/WP106-effectiveness-eu-emissions-trading-system.pdf (accessed on 21 May 2021).

24. Van de Graaf, T.; Colgan, J.D. Russian gas games or well-oiled conflict? Energy security and the 2014 Ukraine crisis. Energy Res. Soc. Sci. 2017, 24, 59-64. [CrossRef]

25. Pollitt, M.G. The European single market in electricity: An economic assessment. Rev. Ind. Organ. 2019, 55, 63-87. [CrossRef]

26. Kuznets, S. Economic growth and income inequality. Amer. Econ. Rev. 1955, 45, 1-28.

27. Halkos, G.; Paizanos, E.A. Exploring the Effect of Economic Growth and Government Expenditure on the Environment; MRNA Paper: Munich, Germany, 2014.

28. Rodrik, D. The Real Exchange Rate and Economic Growth: Theory and Evidence; Harvard University: Cambridge, MA, USA, 2007.

29. Stiglitz, J.E. The Price of Inequality: How Today's Divided Society Endangers Our Future; WW Norton \& Company: New York, NY, USA, 2012.

30. Busch, P.O.; Jörgens, H. The international sources of policy convergence: Explaining the spread of environmental policy innovations. J. Eur. Pub. Policy 2005, 12, 860-884. [CrossRef]

31. Holzinger, K.; Sommerer, T. Race to the bottom or race to Brussels? Environmental competition in Europe. J. Common Mark. Stud. 2011, 49, 315-339. [CrossRef]

32. Holzinger, K.; Knill, C.; Sommerer, T. Environmental policy convergence: The impact of international harmonization, transnational communication, and regulatory competition. Int. Organ. 2008, 62, 553-587. [CrossRef]

33. Holzinger, K.; Knill, C. The interaction of competition, co-operation and communication: Theoretical analysis of different sources of environmental policy convergence. J. Comp. Policy Anal. Res. Pract. 2008, 10, 403-425. [CrossRef]

34. Halkos, G. Environmental Kuznets curve for sulfur: Evidence using GMM estimation and random coefficient panel data models. Environ. Dev. Econ. 2003, 8, 581-601. [CrossRef]

35. Folster, S.; Henrekson, M. Growth effects of government expenditure and taxation in rich countries. Eur. Econ. Rev. 2001, 45, 1501-1520. [CrossRef]

36. Bergh, A.; Karlsson, M. Government size and growth: Accounting for economic freedom and globalization. Public Choice 2010, 142, 195-213. [CrossRef]

37. Grossman, G.; Krueger, A. Economic growth and the environment. Q. J. Econ. 1995, 110, 353-377. [CrossRef]

38. Harbaugh, W.; Levinson, A.; Wilson, D. Re-examining the empirical evidence for an environmental Kuznets curve. Rev. Econ. Stat. 2002, 84, 541-551. [CrossRef]

39. De Bruyn, S.M. Economic Growth and the Environment: An Empirical Analysis; Kluwer Academic Publishers: MA London, UK, 2000.

40. Acemoglu, D.; Robinson, J.A. The political economy of the Kuznets curve. Rev. Dev. Econ. 2002, 6, 183-203. [CrossRef]

41. Stern, D.I. The rise and fall of the environmental Kuznets curve. World Dev. 2004, 32, 1419-1439. [CrossRef]

42. Markandya, A.; Golub, A.; Galinato, S.P. Empirical analysis of national income and $\mathrm{SO}_{2}$ emissions in selected European countries. Environ. Resour. Econ. 2006, 35, 221-257. [CrossRef]

43. Vehmas, J.; Luukkanen, J.; Kaivo-Oja, J. Linking analyses and environmental Kuznets curves for aggregated material flows in the EU. J. Clean. Prod. 2007, 15, 1662-1673. [CrossRef] 
44. Coondoo, D.; Dinda, S. Carbon dioxide emission and income: A temporal analysis of cross-country distributional patterns. Ecol. Econ. 2008, 65, 375-385. [CrossRef]

45. Akbostanc1, E.; Türüt-Asık, S.; Tunç, G.I. The relationship between income and environment in Turkey: Is there an environmental Kuznets curve? Energy Policy 2009, 37, 861-867. [CrossRef]

46. Lee, C.C.; Chiu, Y.-B.; Sun, C.-H. The environmental Kuznets curve hypothesis for water pollution: Do regions matter? Energy Policy 2010, 38, 12-23. [CrossRef]

47. Donfouet, H.P.P.; Jeanty, P.W.; Malin, E. A Spatial Dynamic Panel Analysis of the Environmental Kuznets Curve in European Countries; Center for Research in Economics and Management (CREM): Rennes, France, 2013; Available online: http:/ / crem.univ-rennes1 .fr/wp/2013/201318.pdf (accessed on 12 May 2021).

48. Kaika, D.; Zervas, E. The Environmental Kuznets Curve (EKC) theory-Part A: Concept, causes and the CO2 emissions case. Energy Policy 2013, 62, 1392-1402. [CrossRef]

49. Rafaj, P.; Amann, M.; Siri, J.; Wuester, H. Changes in European greenhouse gas and air pollutant emissions 1960-2010: Decomposition of determining factors. Clim. Chang. 2014, 124, 477-504. [CrossRef]

50. López-Menéndez, A.J.; Pérez, R.; Moreno, B. Environmental costs and renewable energy: Re-visiting the Environmental Kuznets Curve. J. Environ. Manag. 2014, 145, 368-373. [CrossRef] [PubMed]

51. Arbulú, I.; Lozano, J.; Rey-Maquieira, J. Tourism and solid waste generation in Europe: A panel data assessment of the Environmental Kuznets Curve. Waste Manag. 2015, 46, 628-636. [CrossRef] [PubMed]

52. Kasman, A.; Duman, Y.S. $\mathrm{CO}_{2}$ emissions, economic growth, energy consumption, trade and urbanization in new EU member and candidate countries: A panel data analysis. Econ. Model. 2015, 44, 97-103. [CrossRef]

53. Al-Mulali, U.; Tang, C.F.; Ozturk, I. Estimating the Environment Kuznets Curve hypothesis: Evidence from Latin America and the Caribbean countries. Renew. Sustain. Energy Rev. 2015, 50, 918-924. [CrossRef]

54. Shahbaz, M.; Solarin, S.A.; Ozturk, I. Environmental Kuznets Curve hypothesis and the role of globalization in selected African countries. Ecol. Indic. 2016, 67, 623-636. [CrossRef]

55. Bilgili, F.; Koçak, E.; Bulut, Ü. The dynamic impact of renewable energy consumption on $\mathrm{CO}_{2}$ emissions: A revisited Environmental Kuznets Curve approach. Renew. Sustain. Energy Rev. 2016, 54, 838-845. [CrossRef]

56. Dogan, E.; Seker, F. Determinants of $\mathrm{CO}_{2}$ emissions in the European Union: The role of renewable and non-renewable energy. Renew. Energy 2016, 94, 429-439. [CrossRef]

57. Pablo-Romero, M.P.; Cruz, L.; Barata, E. Testing the transport energy-environmental Kuznets curve hypothesis in the EU27 countries. Energy Econ. 2017, 62, 257-269. [CrossRef]

58. Atasoy, B.S. Testing the environmental Kuznets curve hypothesis across the U.S.: Evidence from panel mean group estimators. Renew. Sustain. Energy Rev. 2017, 77, 731-747. [CrossRef]

59. Hanif, I.; Gago-De-Santos, P. The importance of population control and macroeconomic stability to reducing environmental degradation: An empirical test of the environmental Kuznets curve for developing countries. Environ. Dev. 2017, 23, 1-9. [CrossRef]

60. Ahmad, N.; Du, L.; Lu, J.; Wang, J.; Li, H.-Z.; Hashmi, M.Z. Modelling the $\mathrm{CO}_{2}$ emissions and economic growth in Croatia: Is there any environmental Kuznets curve. Energy 2017, 123, 164-172. [CrossRef]

61. Moutinho, V.; Varum, C.; Madaleno, M. How economic growth affects emissions? An investigation of the environmental Kuznets curve in Portuguese and Spanish economic activity sectors. Energy Policy 2017, 106, 326-344. [CrossRef]

62. Pal, D.; Mitra, S.K. The environmental Kuznets curve for carbon dioxide in India and China: Growth and pollution at crossroad. J. Policy Model. 2017, 39, 371-385. [CrossRef]

63. Özokcu, S.; Özdemir, Ö. Economic growth, energy, and environmental Kuznets curve. Renew. Sustain. Energy Rev. 2017, 72, 639-647. [CrossRef]

64. Sinha, A.; Shahbaz, M. Estimation of Environmental Kuznets Curve for $\mathrm{CO}_{2}$ emission: Role of renewable energy generation in India. Renew. Energy 2018, 119, 703-711. [CrossRef]

65. Kiliç, C.; Balan, F. Is there an environmental Kuznets inverted-U shaped curve? Panoeconomicus 2018, 65, 79-94. [CrossRef]

66. Pata, U.K. Renewable energy consumption, urbanization, financial development, income and $\mathrm{CO}_{2}$ emissions in Turkey: Testing EKC hypothesis with structural breaks. J. Clean. Prod. 2018, 187, 770-779. [CrossRef]

67. Aruga, K. Investigating the Energy-Environmental Kuznets Curve hypothesis for the Asia-Pacific region. Sustainability 2019, 11, 2395. [CrossRef]

68. Koilo, V. Evidence of the Environmental Kuznets Curve: Unleashing the opportunity of industry 4.0 in emerging economies. J. Risk Financ. Manag. 2019, 12, 122. [CrossRef]

69. Maneejuk, P.; Yamaka, W.; Sriboonchitta, S. Does the Kuznets curve exist in Thailand? A two decades' perspective (1993-2015). Ann. Oper. Res. 2019, 300, 545-576. [CrossRef]

70. Maneejuk, N.; Ratchakom, S.; Maneejuk, P.; Yamaka, W. Does the Environmental Kuznets Curve exist? An international study. Sustainability 2020, 12, 9117. [CrossRef]

71. Martínez-Navarro, D.; Amate-Fortes, I.; Guarnido-Rueda, A. Inequality and development: Is the Kuznets curve in effect today? Econ. Politica 2020, 37, 703-735. [CrossRef] 
72. Halliru, A.M.; Loganathan, N.; Hassan, A.A.G.; Mardani, A.; Kamyab, H. Re-examining the environmental Kuznets curve hypothesis in the Economic Community of West African States: A panel quantile regression approach. J. Clean. Prod. 2020, 276, 124247. [CrossRef]

73. Shahbaz, M.; Tiwari, K.; Nasir, M. The effects of financial development, economic growth, coal consumption and trade openness on $\mathrm{CO}_{2}$ emissions in South Africa. Energy Policy 2013, 61, 1452-1459. [CrossRef]

74. Liu, X.; Bae, J. Urbanization and industrialization impact of $\mathrm{CO}_{2}$ emissions in China. J. Clean. Prod. 2018, 172, 178-186. [CrossRef]

75. Halkos, G.E.; Paizanos, E.A. The channels of the effect of government expenditure on the environment: Evidence using dynamic panel data. J. Environ. Plann. Manag. 2017, 60, 135-157. [CrossRef]

76. Krajewski, P.; Mackiewicz, M. The role of capital and labour in shaping the environmental effects of fiscal stimulus. J. Clean. Prod. 2019, 216, 323-332. [CrossRef]

77. Hubacek, K.; Baiocchi, G.; Feng, K. Poverty eradication in a carbon constrained world. Nat. Commun. 2017, 8, 912. [CrossRef] [PubMed]

78. Chen, J.D.; Fan, W.; Li, D.; Liu, X.; Song, M.L. Driving factors of global carbon footprint pressure: Based on vegetation carbon sequestration. Appl. Energy 2020, 267, 114914. [CrossRef]

79. Zhang, W.W.; Xu, S.C.; Sharp, B. Do $\mathrm{CO}_{2}$ emissions impact energy use? An assessment of China evidence from 1953 to 2017. China Econ. Rev. 2019, 57, 101340. [CrossRef]

80. Cole, M.A.; Elliott, R.J.R.; Okubo, T.; Zhou, Y. The carbon dioxide emissions of firms: A spatial analysis. J. Environ. Econ. Manag. 2012, 65, 290-309. [CrossRef]

81. Xu, X.K.; Han, L.Y.; Lv, X.F. Household carbon inequality in urban China, its sources and determinants. Ecol. Econ. 2016, 128, 77-86. [CrossRef]

82. Omri, A.; Nguyen, D.K.; Rault, C. Causal interactions between $\mathrm{CO}_{2}$ emissions, FDI, and economic growth: Evidence from dynamic simultaneous-equation models. Econ. Model. 2014, 42, 382-389. [CrossRef]

83. Wang, G.F.; Deng, X.Z.; Wang, J.Y.; Zhang, F.; Liang, S.Q. Carbon emission efficiency in China: A spatial panel data analysis. China Econ. Rev. 2019, 56, 101313. [CrossRef]

84. Rhee, H.C.; Chung, H.S. Change in $\mathrm{CO}_{2}$ emission and its transmissions between Korea and Japan using international input-output analysis. Ecol. Econ. 2006, 58, 788-800. [CrossRef]

85. Cansino, J.M.; Roman, R.; Rueda-Cantuche, J.M. Will China comply with its 2020 carbon intensity commitment? Environ. Sci. Policy 2015, 47, 108-117. [CrossRef]

86. Nag, B.; Parikh, J. Indicators of carbon emission intensity from commercial energy use in India. Energy Econ. 2000, 22, 441-461. [CrossRef]

87. Tan, X.J.; Liu, Y.; Cui, J.B.; Su, B. Assessment of carbon leakage by channels: An approach combining CGE model and decomposition analysis. Energy Econ. 2018, 74, 535-545. [CrossRef]

88. Bekhet, H.A.; Othman, N.S. The role of renewable energy to validate dynamic interaction between $\mathrm{CO}_{2}$ emissions and GDP toward sustainable development in Malaysia. Energy Econ. 2018, 72, 47-61. [CrossRef]

89. Mikayilov, J.I.; Galeotti, M.; Hasanov, F.J. The impact of economic growth on $\mathrm{CO}_{2}$ emissions in Azerbaijan. J. Clean. Prod. 2018, 197, 1558-1572. [CrossRef]

90. Acaravc1, A.; Ozturk, I. On the relationship between energy consumption, $\mathrm{CO} 2$ emissions and economic growth in Europe. Energy 2010, 35, 5412-5420. [CrossRef]

91. Bölük, G.; Mert, M. Fossil \& renewable energy consumption, GHGs (greenhouse gases) and economic growth: Evidence from a panel of EU (European Union) countries. Energy 2014, 74, 439-446.

92. Ahmed, A.; Uddin, G.S.; Sohag, K. Biomass energy, technological progress and the environmental Kuznets curve: Evidence from selected European countries. Biomass-Bioenergy 2016, 90, 202-208. [CrossRef]

93. Abid, M. Does economic, financial and institutional developments matter for environmental quality? A comparative analysis of EU and MEA countries. J. Environ. Manag. 2017, 188, 183-194. [CrossRef] [PubMed]

94. Panayotou, T. Economic Growth and the Environment; Center for International Development (CID) at Harvard University: Cambridge, MA, USA, 2000; Volume 56.

95. Cole, M.A.; Elliott, R.J. Determining the trade-environment composition effect: The role of capital, labor and environmental regulations. J. Environ. Econ. Manag. 2003, 46, 363-383. [CrossRef]

96. Dinda, S. Environmental Kuznets curve hypothesis: A survey. Ecol. Econ. 2004, 49, 431-455. [CrossRef]

97. Tsurumi, T.; Managi, S. Decomposition of the environmental Kuznets curve: Scale, technique, and composition effects. Environ. Econ. Policy Stud. 2010, 11, 19-36. [CrossRef]

98. Menegaki, A.N.; Tsagarakis, K.P. Rich enough to go renewable, but too early to leave fossil energy? Renew. Sustain. Energy Rev. 2015, 41, 1465-1477. [CrossRef]

99. Freeman, A.M. Air and Water Pollution Control: A Benefit-Cost Assessment; Wiley: New York, NY, USA, 1982.

100. Freeman, A.M. Water pollution policy. In Public Policies for Environmental Protection; Portney, P., Ed.; Resources for the Future: Washington, DC, USA, 2000.

101. Hahn, R. (Ed.) Regulatory reform: What the government's numbers tell us. In Risks, Costs and Lives Saved: Getting Better Results from Regulation; Oxford University Press: Oxford, UK, 1996. 
102. Portney, P. Air pollution policy. In Public Policies for Environmental Protection; Resources for the Future: Washington, DC, USA, 2000.

103. Mandl, U.; Dierx, A.; Ilzkovitz, F. The Effectiveness and Efficiency of Public Spending; Directorate General Economic and Financial Affairs (DG ECFIN), European Commission: Luxembourg, 2008; Volume 301.

104. Srebotnjak, T. Encyclopedia of Quantitative Risk Analysis and Assessment; Environmental performance index; John Wiley \& Sons, Ltd.: Singapore, 2008.

105. Apergis, N.; Christou, C.; Hassapis, C. Convergence in public expenditures across EU countries: Evidence from club convergence. Econ. Financ. Res. 2013, 1, 45-59. [CrossRef]

106. Ferreiro, J.; García-del-Valle, M.T.; Gómez, C. Social preferences and fiscal policies: An analysis of the composition of public expenditures in the European Union. J. Post Keynes. Econ. 2010, 32, 347-370. [CrossRef]

107. Ferreiro, J.; García-del-Valle, M.T.; Gómez, C. An analysis of the convergence of the composition of public expenditures in European Union countries. Am. J. Econ. Soc. 2013, 72, 799-825. [CrossRef]

108. Ullah, S.; Majeed, M.T.; Chishti, M.Z. Examining the asymmetric effects of fiscal policy instruments on environmental quality in Asian economies. Environ. Sci. Pollut. Res. 2020, 27, 38287-38299. [CrossRef] [PubMed]

109. Chen, A.P.; Groenewold, N. China's "New Normal": Is the growth slowdown demand-or supply-driven? China Econ. Rev. 2019, 58, 101203. [CrossRef]

110. Cheong, T.S.; Wu, Y.R. The impacts of structural transformation and industrial upgrading on regional inequality in China. China Econ. Rev. 2014, 31, 339-350. [CrossRef]

111. Zhou, C.; Zhang, X. Measuring the Efficiency of Fiscal Policies for Environmental Pollution Control and the Spatial Effect of Fiscal Decentralization in China. Int. J. Environ. Res. Public Health 2020, 17, 8974. [CrossRef]

112. Farrell, M.J. The measurement of productive efficiency. J. R. Stat. Soc. Ser. A 1957, 120, 253-290. [CrossRef]

113. Charnes, A.; Cooper, W.W.; Rhodes, E. Measuring the efficiency of decision making units. Eur. J. Oper. Res. 1979, 2, 429-444. [CrossRef]

114. Cooper, W.W.; Seiford, L.M.; Tone, K. Data Envelopment Analysis: A Comprehensive Text with Models, Applications, References and DEA-Solver Software; Kluwer Academic Publishers: Boston, MA, USA, 2000.

115. Cook, W.D.; Tone, K.; Zhu, J. Data envelopment analysis: Prior to choosing a model. Omega 2014, 44, 1-4. [CrossRef]

116. Jenkins, L.; Anderson, M. A multivariate statistical approach to reducing the number of variables in data envelopment analysis. Eur. J. Oper. Res. 2003, 147, 51-61. [CrossRef]

117. Thanassoulis, E. Using data envelopment analysis in practice. In Introduction to the Theory and Application of Data Envelopment Analysis; Kluwer Academic Publishers: Norwell, MA, USA, 2001; pp. 89-121. [CrossRef]

118. Cooper, J.O.; Heron, T.E.; Heward, W.L. Applied Behavior Analysis, 2nd ed.; Pearson: Upper Saddle River, NJ, USA, 2007.

119. Simar, L.; Wilson, P. Non-parametric tests of returns to scale. Eur. J. Oper. Res. 2002, 139, 115-132. [CrossRef]

120. Huguenin, J. Data Envelopment Analysis (DEA): A Pedagogical Guide for Decision Makers in the Public Sector; Swiss Graduate School of Public Administration: Lausanne, Switzerland, 2012.

121. Alirezaee, M.R.; Howland, M.; van de Panne, C. Sampling size and efficiency bias in data envelopment analysis. J. Appl. Math. Decis. Sci. 1998, 2, 51-64. [CrossRef]

122. Dobrzanski, P. Spending on innovation as a factor of competitiveness of market economies. In Country Experiences in Economic Development, Management and Entrepreneurship; Bilgin, M., Danis, H., Demir, E., Can, U., Eds.; Springer: Cham, Switzerland, 2017; Volume 5, pp. 125-137. [CrossRef]

123. Wober, K.W. Data envelopment analysis. J. Travel Tour. Mark. 2007, 21, 91-108. [CrossRef]

124. Zhang, Y.; Bartels, R. The effect of sample size on the mean efficiency in DEA with an application to electricity distribution in Australia, Sweden and New Zealand. J. Prod. Anal. 1998, 9, 187-204. [CrossRef]

125. Banker, R.D.; Charnes, A.; Cooper, W.W.; Swarts, J.; Thomas, D. An Introduction to Data Envelopment Analysis with Some of Its Models and Their Uses; Research Government and Nonprofit Accounting; JAI Press: Greenich, CT, USA, 1989; Volume 5, pp. $125-163$.

126. Eurostat. National Expenditure on Environmental Protection. Available online: https://appsso.eurostat.ec.europa.eu/nui/ submitViewTableAction.do (accessed on 11 June 2021).

127. World Bank, Environment. Available online: https:/ / data.worldbank.org/topic/environment (accessed on 11 June 2021).

128. Cai, Y. Factors Affecting the Efficiency of the BRICS' National Innovation Systems: A Comparative Study Based on DEA and Panel Data Analysis; Economics (Open e-Journal), Economics Discussion Papers; Kiel Institute for the World Economy: Kiel, Germany, 2011; Volume 52.

129. Podinovski, V. Production trade-offs and weight restrictions in data envelopment analysis. J. Oper. Res. Soc. 2004, 55, 1311-1322. [CrossRef]

130. European Commission. SF 2007-2013 Funds Absorption Rate. Available online: https:/ / cohesiondata.ec.europa.eu/2007-2013Finances/SF-2007-2013-Funds-Absorption-Rate/kk86-ceun/data (accessed on 11 June 2021).

131. European Environment Agency. The European Environment-State and Outlook 2020. Knowledge for Transition to A Sustainable Europe; Publications Office of the European Union: Luxembourg, 2019.

132. Berg, A.; Buffie, E.F.; Pattillo, C.; Portillo, R.; Presbitero, A.; Zanna, L.-F. Some Misconceptions about Public Investment Efficiency and Growth; IMF Working Paper 15/272; International Monetary Fund: Washington, DC, USA, 2015. 
133. Shen, W.; Yang, S.-C.S.; Zanna, L.-F. Government spending effects in low-income countries. J. Dev. Econ. 2018, 133, $201-219$. [CrossRef]

134. European Anti-Fraud Office. The OLAF Report 2020; Publications Office of the European Union: Luxembourg, 2021. 